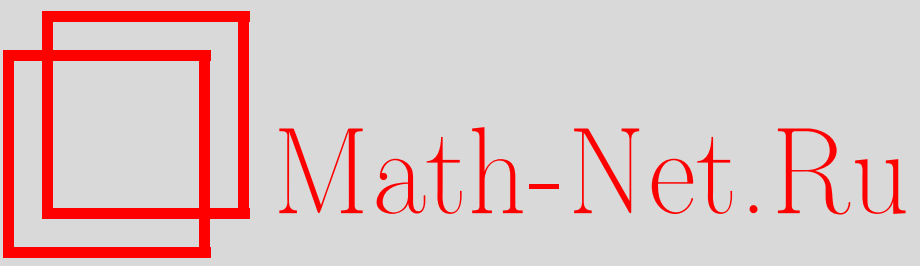

В. В. Пупышев, Строение регулярных решений трехчастичного уравнения Шредингера вблизи точки парного удара, ТМФ, 2003, том 136, номер 1, 90-114

DOI: https://doi.org/10.4213/tmf216

Использование Общероссийского математического портала Math-Net.Ru подразумевает, что вы прочитали и согласны с пользовательским соглашением

http://www.mathnet.ru/rus/agreement

Параметры загрузки:

IP: 54.224 .187 .69

26 апреля 2023 г., 16:27:39 


\section{СТРОЕНИЕ РЕГУЛЯРНЫХ РЕШЕНИЙ ТРЕХЧАСТИЧНОГО УРАВНЕНИЯ ШРЕДИНГЕРА ВБЛИЗИ ТОЧКИ ПАРНОГО УДАРА}

Исследуется шестимерное уравнение Шредингера для системы трех частиц с центральными парными взаимодействиями более общего вида, чем кулоновские. Регулярные общее и частные физические решения такого уравнения представлены бесконечными асимптотическими рядами по целым степеням расстояния между двумя частицами и искомым функциям других трехчастичных координат. Построение таких функций в угловых базисах, образованных сферическими и бисферическими гармониками или симметризованными $D$-функциями Вигнера, сведено к решению простых алгебраических рекуррентных уравнений. Для проекций физических решений на угловые базисные функции выведены граничные условия в точке парного удара.

Ключевые слова: задача трех частиц, уравнение Шредингера, регулярные решения, асимптотические разложения.

\section{1. ВВЕДЕНИЕ}

Приведем основные определения. Будем использовать систему единиц, в которой заряд электрона $e$ и постоянная Планка $\hbar$ равны единице. В трехмерном координатном пространстве $\mathcal{R}^{3}$ фиксируем декартову систему координат $S$ с ортами $\hat{\mathbf{e}}_{1}, \hat{\mathbf{e}}_{2}$ и $\hat{\mathbf{e}}_{3}$ и начальной точкой $O$, совпадаюшей с центром масс исследуемой системы $\left(p_{1}, p_{2}, p_{3}\right)$ трех частиц $p_{1}, p_{2}$ и $p_{3}$ с массами $m_{1}, m_{2}, m_{3}$ и зарядами $z_{1}, z_{2}, z_{3}$. Пусть в этой системе $\mathbf{a}_{i j}-$ разность радиус-векторов $\mathbf{a}_{i}$ и $\mathbf{a}_{j}$ частиц $p_{i}$ и $p_{j}$, а $\mathbf{x}_{k}$ и $\mathbf{y}_{k}-$ приведенные векторы Якоби [1],

$$
\begin{aligned}
\mathbf{x}_{k} & \equiv \sqrt{2 \mu_{i j}}\left(\mathbf{a}_{j}-\mathbf{a}_{i}\right), & \mu_{i j} & \equiv \frac{m_{i} m_{j}}{m_{i}+m_{j}}, \\
\mathbf{y}_{k} & \equiv \sqrt{2 \mu_{k, i j}}\left(\frac{m_{i} \mathbf{a}_{i}+m_{j} \mathbf{a}_{j}}{m_{i}+m_{j}}-\mathbf{a}_{k}\right), & \mu_{k, i j} & \equiv \frac{m_{k}\left(m_{i}+m_{j}\right)}{m_{1}+m_{2}+m_{3}}
\end{aligned}
$$

где индексы $i, j, k$ образуют циклическую перестановку индексов $1,2,3:$ индекс $i$ переходит в $k, j-$ в $i$, а $k$-в $j$. В шестимерном координатном пространстве $\mathcal{R}^{6}$ векторам $\mathbf{x}_{k}$

* Объединенный институт ядерных исследований, Дубна, Московская обл., Россия. E-mail: pupyshev@thsun1.jinr.ru 
и $\mathbf{y}_{k}$ сопоставим вектор $\mathbf{r}_{k}=\left(\mathbf{x}_{k}, \mathbf{y}_{k}\right)$. Выберем пару $\left(p_{j}, p_{k}\right)$ частиц $p_{j}$ и $p_{k}$. Пусть $R-$ расстояние между ними, $\mathbf{R} \equiv \mathbf{a}_{j k} \uparrow \uparrow \mathbf{x}_{i}$, а $\rho$ - расстояние от оставшейся частищы $p_{i}$ до центра масс этой пары, $\boldsymbol{\rho} \uparrow \mathbf{y}_{i}$. В $\mathcal{R}^{6}$ под окрестностью точки парного удара частиц $p_{j}$ и $p_{k}$ подразумевается шестимерная область $\mathcal{G}$, в которой эти частицы близки друг к другу, но отделены от частицы $p_{i}$,

$$
\mathcal{G} \equiv\left\{\mathbf{r} \equiv \mathbf{r}_{i}: R \ll 1, \rho>0\right\}=\left\{\mathbf{r}: x \equiv x_{i} \ll 1, y \equiv y_{i}>0\right\}
$$

Пусть $\Psi$ - общее регулярное $(|\Psi(\mathbf{r})|<\infty \forall \mathbf{r})$ решение уравнения Шредингера для системы $\left(p_{1}, p_{2}, p_{3}\right)$ в $\mathcal{R}^{6}$,

$$
(H-E) \Psi=0, \quad H=H_{0}+V, \quad V \equiv \sum_{k=1}^{3} V_{k}
$$

где $H_{0}$ - свободный гамильтониан, $E$ - полная энергия, $V_{k}$ - взаимодействие между частицами $p_{i}$ и $p_{j}$. Явный вид решения $\Psi$, как правило, неизвестен. Поэтому судить о его поведении в физически интересных областях пространства $\mathcal{R}^{6}$ можно лишш по асимптотическим разложениям.

Вывод и анализ асимптотических разложений общего регулярного решения $\Psi$ представляется теоретически важным и интересным. Зная такие разложения, можно легко найти разложение любого регулярного частного решения, например, трехчастичной волновой функции $\Psi^{\varepsilon}$, обладающей (в отличие от $\Psi$ ) полным набором $\varepsilon$ сохраняющихся квантовых чисел. Вывод разложения для $\Psi^{\varepsilon}$ сводится к проецированию найденного разложения для $\Psi$ на базис из собственных функций всех операторов, коммутирующих с полным гамильтонианом $H$.

Стоит отметить, что замена функции $\Psi^{\varepsilon}$ в уравнениях Фаддеева [1]

$$
\left(H_{0}-E\right) \Psi_{k}^{\varepsilon}=-V_{k} \Psi^{\varepsilon}, \quad \Psi^{\varepsilon}=\Psi_{1}^{\varepsilon}+\Psi_{2}^{\varepsilon}+\Psi_{3}^{\varepsilon}
$$

полученным для нее разложением открывает способ построения разложений фаддеевских компонент $\Psi_{k}^{\varepsilon}$, отличный от предложенного в работе [2].

В случае центральных парных взаимодействий, например, кулоновских

$$
V_{k}\left(x_{k}\left(a_{i j}\right)\right)=\frac{z_{i} z_{j}}{a_{i j}}, \quad V_{k}\left(x_{k}\right)=\frac{q_{k}}{x_{k}}, \quad q_{k} \equiv z_{i} z_{j} \sqrt{2 \mu_{i j}}, \quad k=1,2,3,
$$

или взаимодействий более общего вида

$$
V_{k}\left(x_{k}\right)=\frac{q_{k}}{x_{k}}+\bar{V}_{k}\left(x_{k}\right), \quad \bar{V}_{k}\left(x_{k}\right)=\sum_{n=0}^{\infty} x_{k}^{n} \bar{V}_{k n}, \quad \bar{V}_{k n}=\text { const }, \quad k=1,2,3,
$$

с гамильтонианом $H$ коммутируют квадрат оператора полного углового момента $\mathbf{1}$, его компонента $l_{3}$ и оператор $P_{r}$ инверсии $\mathbf{r} \rightarrow-\mathbf{r}$. Набор $\varepsilon=(E, \ell, m, \sigma)$ состоит из собственных чисел этих операторов. В качестве соответствуюших собственных функций 
часто используются бисферические гармоники $\mathcal{Y}_{a b}^{\ell m}$ и симметризованные линейные комбинации $D$-функций Вигнера $D_{m m^{\prime}}^{\ell}$. Бисферические гармоники выражаются через коэффоциенты Клебша-Гордана $C_{a \alpha b \beta}^{\ell m}$ и сферические функции

$$
Y_{f e}(\hat{a})=\frac{1}{\sqrt{2 \pi}} e^{-i e \varphi_{a}} \Theta_{f e}\left(\cos \theta_{a}\right), \quad \hat{a} \equiv\left(\theta_{a}, \varphi_{a}\right), \quad \hat{a}=\hat{x}, \hat{y},
$$

формулами [3], [4]

$$
\mathcal{Y}_{a b}^{\ell m}(\hat{x}, \hat{y}) \equiv \sum_{\alpha=-a}^{a} C_{a \alpha b \beta}^{\ell m} Y_{a \alpha}(\hat{y}) Y_{b \beta}(\hat{x}), \quad \mathbf{a}+\mathbf{b}=1, \quad(-1)^{a+b}=\sigma,
$$

а симметризованные $D$-функции можно представить в виде [5]

$$
D_{m m^{\prime}}^{\ell \sigma}\left(\omega^{t}\right)=\left[\frac{2 \ell+1}{16 \pi^{2}\left(1+\delta_{m^{\prime} 0}\right)}\right]^{1 / 2}\left[D_{m m^{\prime}}^{\ell}\left(\omega^{t}\right)+\sigma(-1)^{\ell-m^{\prime}} D_{m,-m^{\prime}}^{\ell}\left(\omega^{t}\right)\right]
$$

где $\omega^{t}=\left(\alpha^{t}, \beta^{t}, \gamma^{t}\right)$ - набор углов Эйлера, определяюших ориентацию выбранной “подвижной" системы координат $S^{t}$ относительно системы $S$. Например, в работах [6], [7] использовалась система $S^{t}, t=R$, с ортом $\hat{\mathbf{e}}_{3}^{R}$, коллинеарным вектору $\mathbf{R}$, соединяющему частицы $p_{1}$ и $p_{2}$, и ортом $\hat{\mathbf{e}}_{1}^{R}$, расположенным в плоскости трех частиц и направленным к частице $p_{3}$.

Знание асимптотических разложений волновой функции $\Psi^{\varepsilon}$ необходимо для вычисления с высокой точностью ее приближения $\widetilde{\Psi}^{\varepsilon}$ и, следовательно, для последуюшего достоверного определения всех наблюдаемых величин. Дело в том, что учет всех особенностей поведения искомого решения дифференциального уравнения (в нашем случае $\left.\Psi^{\varepsilon}\right)$ улучшает поточечную сходимость любого численного метода [8]. В [6], [7] было отмечено, что такой учет поведения $\Psi^{\varepsilon}$ в области $\mathcal{G}$ особенно важен и эффективен при реализации метода Ритца [8], [9], когда координатную зависимость опорных функций $F_{n}^{\varepsilon}$ вариационного анзаца $\widetilde{\Psi}^{\varepsilon} \approx F_{1}^{\varepsilon}+F_{2}^{\varepsilon}+\cdots$ приходится определять заранее и всюду в пространстве $\mathcal{R}^{6}$.

C другой стороны, такие наблюдаемые характеристики трехчастичных систем, как релятивистские поправки, $\gamma$-факторы, коэффициенты рекомбинации и сечения астрофизических ядерных процессов [10], выражаются через интегралы, содержащие волновую функцию $\widetilde{\Psi}^{\varepsilon}$. Поэтому точность вычисления таких характеристик тем выше, чем лучше аппроксимация $\Psi^{\varepsilon} \approx \Psi$ во всей области $\mathcal{G}$.

В подходе Ритца, в вариационно-разностных и проекционно-сеточных схемах [8] и в методе сплайн-коллокаций [9], [11] задачу такого приближения можно решить, подчинив частные производные искомой функции $\widetilde{\Psi}^{\varepsilon}$ или ее проекций на базисы $(6)-(8)$ тем же линейным граничным условиям (связям при $x=0, y>0)$, которым удовлетворяют частные производные точного решения $\Psi^{\varepsilon}$ уравнения Шредингера $(2)$ или соответствующие проекции этого решения. Примером такой связи может служить соотношение

$$
\sum_{n=0}^{n^{\prime}<\infty} A_{n}(\hat{x}, \mathbf{y}) \partial_{x}^{n} \Psi^{\varepsilon}(\mathbf{x}, \mathbf{y})=0, \quad x=0, \quad y>0
$$


где $A_{n}$ - известные функции или линейные комбинации известных функций и операторов частных производных по аргументам $\hat{x}$ и у. Знание связей типа $(9)$ позволяет решить и обратную задачу: по величине невязки

$$
\chi=\sum_{n=0}^{n^{\prime}<\infty} A_{n}(\hat{x}, \mathbf{y}) \partial_{x}^{n} \widetilde{\Psi}^{\varepsilon}(\mathbf{x}, \mathbf{y}), \quad x=0, \quad y>0,
$$

оценить, насколько приближенное решение $\widetilde{\Psi}^{\varepsilon}$, полученное каким-то способом, близко к точному решению $\Psi$ в окрестности точки парного удара.

Для примера выведем связи из известных асимптотик функции $\Psi^{\varepsilon}$. Под проекцией $\langle f(\xi) \mid g\rangle$ функции $g$ на функцию $f(\xi)$ будем подразумевать интеграл от произведения $f^{*}(\xi) g$ по всей области изменения аргументов $\xi$.

Като [12] впервые показал, что $S$-волновая $(\ell=0)$ функция $\Psi^{\varepsilon}\left(a_{1}, a_{2}, a_{12}\right)$ двух электронов $p_{1}$ и $p_{2}$ в кулоновском поле бесконечно тяжелого $\left(m_{3}=\infty\right)$ ядра $p_{3}$ подчиняется нелокальному граничному условию

$$
\partial_{a}\left\langle\Psi^{\varepsilon}\left(a_{1}, a_{2}, a_{12}\right)\right\rangle_{\delta}=\lambda \Psi^{\varepsilon}\left(a_{1}, a_{2}, a_{12}\right), \quad a=0
$$

где $\left\langle\Psi^{\varepsilon}\right\rangle_{\delta}$ - среднее по сфере бесконечно малого радиуса $\delta$ с центром в точке $a=0 ; \lambda=$ $m_{1} z_{3}$ при $a=a_{i}, i=1,2$, и $\lambda=\mu_{12}=m_{1} / 2$ при $a=a_{12}$.

Позже в работе [13] для той же волновой функции было получено асимптотическое разложение

$$
\Psi^{\varepsilon}\left(a_{1}, a_{2}, a_{12}\right)=\left(1+m_{1} z_{3} a_{1}\right) \Psi^{\varepsilon}\left(0, a_{2}, a_{12}\right)+\left(\mathbf{a}_{1} \mathbf{A}\right)+O\left(a_{1}^{2}\right), \quad a_{1} \rightarrow 0,
$$

где $\mathbf{A}$ - неопределенный вектор из $\mathcal{R}^{3}$. Проекция $\Psi_{00}^{\varepsilon} \equiv\left\langle Y_{00}\left(\hat{a}_{1}\right) \mid \Psi^{\varepsilon}\right\rangle$ на сферическую функцию $Y_{00}\left(\hat{a}_{1}\right)$ не содержит $\mathbf{A}$ и удовлетворяет связи типа $(9)$,

$$
\left(\partial_{a_{1}}-m_{1} z_{3}\right) \Psi_{00}^{\varepsilon}\left(a_{1}, a_{2}, a_{12}\right)=0, \quad a_{1}=0, \quad a_{2}>0 .
$$

Авторы работы [14] записали $N$-частичное уравнение Шредингера с кулоновскими взаимодействиями в асимптотическом виде

$$
\left[-\frac{\Delta_{R}}{2 \mu_{12}}+\frac{z_{1} z_{2}}{R}+O(1)\right] \Psi=0, \quad R=a_{12} \rightarrow 0,
$$

что позволило им определить асимптотику обшего решения $\Psi$, но лишш с точностью порядка $O\left(R^{2}\right)$. В случае $N=3$ такая асимптотика имеет вид

$$
\Psi=f_{00}^{0} Y_{00}(\widehat{R})\left(1+\mu_{12} z_{1} z_{2} R\right)+R \sum_{\beta=-1}^{1} f_{1 \beta}^{1} Y_{1 \beta}(\widehat{R})+O\left(R^{2}\right), \quad R \rightarrow 0
$$

Если ее продифференцировать по $R$ и результат спроецировать на функцию $Y_{00}(\widehat{R})$, то для проекции $\Psi_{00} \equiv\left\langle Y_{00}(\widehat{R}) \mid \Psi\right\rangle$ получится связь, не содержашая неопределенных и не зависящих от $R$ множителей $f_{00}^{0}$ и $f_{1 \beta}^{1}$,

$$
\left(\partial_{R}-\mu_{12} z_{1} z_{2}\right) \Psi_{00}=0, \quad R=0 .
$$


В работе [6] для $D$-компонент $\Psi_{m^{\prime}}^{\varepsilon R}$ трехчастичной волновой функции

$$
\Psi^{\varepsilon}(\mathbf{R}, \boldsymbol{\rho})=\sum_{m^{\prime}=0}^{\ell} D_{m m^{\prime}}^{\ell \sigma *}\left(\omega^{R}\right) \Psi_{m^{\prime}}^{\varepsilon R}(R, \rho, \theta), \quad \cos \theta=\frac{\mathbf{R} \boldsymbol{\rho}}{R \rho}, \quad \sigma=(-1)^{\ell}
$$

в случае кулоновских взаимодействий (4) была доказана асимптотическая формула

$$
\begin{aligned}
\Psi_{m^{\prime}}^{\varepsilon}(R, \rho, \theta)= & f_{\ell m^{\prime}}^{0}(\rho) Y_{\ell m^{\prime}}(\theta, 0)\left(1+\mu_{12} z_{1} z_{2} R\right)+ \\
& +R \sum_{a=|\ell \pm 1|} f_{a m^{\prime}}^{1}(\rho) Y_{a m^{\prime}}(\theta, 0)+O\left(R^{2}\right), \quad R \rightarrow 0, \quad \rho>0
\end{aligned}
$$

Дифференцируя (13) по $R$ и проецируя полученное равенство на функции $\Theta_{\ell m^{\prime}}(\theta)$, исключаем все неизвестные функций $f_{\ell m^{\prime}}^{0}, f_{a m^{\prime}}^{1}$ и приходим к связям

$$
\left(\partial_{R}-\mu_{12} z_{1} z_{2}\right) \Psi_{\ell m^{\prime}}^{\varepsilon R}(R, \rho)=0, \quad \Psi_{\ell m^{\prime}}^{\varepsilon R}(R, \rho) \equiv\left\langle\Theta_{\ell m^{\prime}}(\theta) \mid \Psi_{m^{\prime}}^{\varepsilon R}\right\rangle, \quad R=0, \quad \rho>0 .
$$

Из формул (11)-(13) нельзя получить никаких других связей типа (9), потому что явный вид слагаемых, убывающих как $O\left(R^{2}\right)$, неизвестен. Для вывода граничных условий, связывающих производные решений $\Psi$ или $\Psi^{\varepsilon}$ более высокого порядка, необходимо знать в области $\mathcal{G}$ их полные асимптотические разложения (бесконечные ряды). Выводи анализ таких разложений в случае центральных взаимодействий более общего вида (5), чем кулоновские взаимодействия (4), составляет основное содержание данной работы.

\section{2. РЯДЫ ПАРНЫХ И ПОЛНОГО ВЗАИМОДЕЙСТВИЙ}

Пусть некулоновские слагаемые $\bar{V}_{k}$ парных взаимодействий (5) являются аналитическими функциями. Будем далее полагать

$$
x \equiv x_{i} \rightarrow 0, \quad y \equiv y_{i}>0, \quad u \equiv \cos \theta=\frac{\mathbf{x y}}{x y}, \quad k \neq i, \quad q \equiv q_{i},
$$

и использовать координатное представление $\langle\mathbf{x}, \mathbf{y}| \equiv\left\langle\mathbf{x}_{i}, \mathbf{y}_{i}\right|$.

Так как векторы Якоби (1) кинематически связаны [15],

$$
\left(\begin{array}{l}
\mathbf{x}_{k} \\
\mathbf{y}_{k}
\end{array}\right)=-\left(\begin{array}{cc}
+c_{k i} & s_{k i} \\
-s_{k i} & c_{k i}
\end{array}\right)\left(\begin{array}{c}
\mathbf{x}_{i} \\
\mathbf{y}_{i}
\end{array}\right), \quad\left(\begin{array}{c}
c_{k i} \\
s_{k i}
\end{array}\right) \equiv\left(\begin{array}{c}
\cos \gamma_{k i} \\
\sin \gamma_{k i}
\end{array}\right), \quad \gamma_{k i} \in\left[-\frac{\pi}{2}, \frac{\pi}{2}\right],
$$

где $\gamma_{k i}$ - кинематический угол, зависящий только от отношений масс частиц, то $x_{k}-$ функция аргументов $x, y, u$,

$$
\left\langle\mathbf{x}, \mathbf{y} \mid x_{k}\right\rangle=x_{k}(x, y, u)=\frac{\left|s_{k i}\right| y}{g(v)}, \quad g(v) \equiv\left(1-2 u v+v^{2}\right)^{-1 / 2}, \quad v \equiv-\frac{x c_{k i}}{y s_{k i}},
$$

а функция $1 / x_{k}$ пропорциональна производяшей функции $g(v)$ для полиномов Лежандра $P_{n}(u)$ [3]. Поэтому слагаемые $q_{k} / x_{k}$ в суммах (5) представляют собой ряды

$$
\frac{q_{k}}{x_{k}}=\frac{q_{k}}{\left|s_{k i}\right| y} \sum_{n=0}^{\infty}\left(-\frac{c_{k i} x}{s_{k i} y}\right)^{n} P_{n}(u), \quad k \neq i .
$$


Из соотношений (14) и (15) вытекает формула дифференцирования

$$
\left.\partial_{x}^{n} x_{k}\right|_{x=0}=c_{k i} \frac{s_{k i}}{\left|s_{k i}\right|} \frac{n !}{2 n-1}\left(-\frac{c_{k i}}{s_{k i} y}\right)^{n-1}\left[P_{n}(u)-P_{n-2}(u)\right], \quad n=0,1, \ldots,
$$

откуда следует, что каждый член $T_{p}$ ряда Тейлора функции $\bar{V}_{k}\left(x_{k}(x, y, u)\right)$ с центром в точке $x=0$ записывается в виде суммы по полиномам $P_{s}(u)$ с индексом $s \leqslant p$,

$$
T_{p}(y, u)=\left.\frac{x^{p}}{p !} \partial_{x}^{p} \bar{V}_{k}\left(x_{k}(x, y, u)\right)\right|_{x=0}=\frac{x^{p}}{p !} \sum_{s=0}^{p} \bar{V}_{k}^{p s}\left(\left|s_{k i}\right| y\right) P_{s}(u)
$$

Если $P_{x}$ и $P_{u}-$ операторы инверсии $x \rightarrow-x$ и $u \rightarrow-u$, то

$P_{u} P_{s}(u)=(-1)^{s} P_{s}(u), \quad P_{x} x^{p}=(-1)^{p} x^{p}, \quad P_{x} P_{u} x_{k}=x_{k}, \quad\left(1-P_{x} P_{u}\right) V_{k}\left(x_{k}\right)=0$.

Из этих соотношений и линейной независимости функций $x^{0}, x^{1}, \ldots$ следует, что $P_{x} P_{u} T_{p}=T_{p}$ для всех $p$. Поэтому ряд Тейлора для $\bar{V}_{k}$ сводится к сумме по индексам $p$ и $s$ таким, что $p+s$-четное число:

$$
\bar{V}_{k}\left(x_{k}(x, y, u)\right)=\sum_{p=0}^{\infty} x^{p} \sum_{s=0}^{p} \bar{V}_{k}^{p s}\left(\left|s_{k i}\right| y\right) P_{s}(u), \quad(-1)^{p+s}=1 .
$$

Вследствие представления (5) для $\bar{V}_{i}$ и представлений $(15),(16)$ полное взаимодействие $V=V_{1}+V_{2}+V_{3}$ разлагается в двойной ряд

$$
\begin{aligned}
V(x, y, u) & =\frac{q}{x}+V^{00}(y)+\sum_{p=1}^{\infty} x^{p} \sum_{s=0}^{p} V^{p s}(y) P_{s}(u), \quad q \equiv q_{i}, \\
V^{p s}(y) & \equiv \bar{V}_{i p} \delta_{s 0}+\delta_{p s} \sum_{k \neq i} \frac{q_{k}}{\left|s_{k i}\right| y}\left(-\frac{c_{k i}}{s_{k i} y}\right)^{p}+\sum_{k \neq i} \bar{V}_{k}^{p s}(y),
\end{aligned}
$$

где отличны от нуля только константы $V^{p 0}=\bar{V}_{i p}$ с нечетным $p$ и функции $V^{p s}(y)$ с четной суммой $p+s$. При $p \leqslant 2$ их можно найти по формулам

$$
\begin{aligned}
V^{00}(y) & =\bar{V}_{i 0}+\sum_{k \neq i}\left[q_{k}\left(\left|s_{k i}\right| y\right)^{-1}+\bar{V}_{k}\left(\left|s_{k i}\right| y\right)\right], \quad V^{10}(y)=\bar{V}_{i 1}, \\
V^{11}(y) & =\sum_{k \neq i} c_{k i}\left(s_{k i}\left|s_{k i}\right|^{-1}\right)\left[\bar{V}_{k}^{\prime}\left(\left|s_{k i}\right| y\right)-q_{k}\left(s_{k i} y\right)^{-2}\right], \\
V^{20}(y) & =\bar{V}_{i 2}+g^{-}(y), \quad V^{22}(y)=\sum_{k \neq i} q_{k} c_{k i}^{2}\left(\left|s_{k i}\right| y\right)^{-3}+g^{+}(y), \\
g^{ \pm}(y) & \equiv \frac{1}{6} \sum_{k \neq i} c_{k i}^{2}\left[\bar{V}_{k}^{\prime \prime}\left(\left|s_{k i}\right| y\right) \pm 2 \bar{V}_{k}^{\prime}\left(\left|s_{k i}\right| y\right)\left(\left|s_{k i}\right| y\right)^{-1}\right],
\end{aligned}
$$

где $\bar{V}_{k}^{\prime}$ и $\bar{V}_{k}^{\prime \prime}-$ первая и вторая производные функции $\bar{V}_{k}\left(x_{k}\right)$ по аргументу $x_{k}$ в точке $x_{k}=\left|s_{k i}\right| y$, т.е. при $x=0$. 
Для проецирования ряда (17) потребуются матричные элементы полинома $P_{s}$ в базисах (6) и (7). Если использовать известные формулы [3]

$$
\begin{gathered}
P_{s}(u)=\frac{4 \pi}{2 s+1} \sum_{\alpha=-s}^{s} Y_{s \alpha}^{*}(\hat{y}) Y_{s \alpha}(\hat{x}), \\
\left\langle Y_{c \gamma}(\hat{x}) \mid Y_{a \alpha}(\hat{x}) Y_{b \beta}(\hat{x})\right\rangle=(-1)^{a}\left(\frac{2 a+1}{4 \pi}\right)^{1 / 2} C_{a 0 c 0}^{b 0} C_{a \alpha b \beta}^{c \gamma}
\end{gathered}
$$

и представление (6), то нетрудно получить искомые выражения

$$
\begin{aligned}
\left\langle Y_{b \beta}(\hat{x})\left|P_{s}(u)\right| Y_{b^{\prime} \beta^{\prime}}(\hat{x})\right\rangle= & (-1)^{s}\left(\frac{4 \pi}{2 s+1}\right)^{1 / 2} C_{s 0 b 0}^{b^{\prime} 0} C_{s \alpha b \beta^{\prime}}^{b \beta} Y_{s \alpha}^{*}(\hat{y}), \\
\left\langle\mathcal{Y}_{a b}^{\ell m}(\hat{x}, \hat{y})\left|P_{s}(u)\right| \mathcal{Y}_{a^{\prime} b^{\prime}}^{\ell m}(\hat{x}, \hat{y})\right\rangle= & (-1)^{a+b-\ell+s} C_{s 0 a^{\prime} 0}^{a 0} C_{s 0 b^{\prime} 0}^{b 0} \times \\
& \times\left[\left(2 a^{\prime}+1\right)\left(2 b^{\prime}+1\right)\right]^{1 / 2}\left\{\begin{array}{ccc}
a & a^{\prime} & s \\
b^{\prime} & b & \ell
\end{array}\right\}, \\
\left\langle\Theta_{b \beta}(u)\left|P_{s}(u)\right| \Theta_{b^{\prime} \beta^{\prime}}(u)\right\rangle= & \delta_{\beta \beta^{\prime}}(-1)^{s}\left(\frac{2 s+1}{2}\right)^{1 / 2} C_{s 0 b 0}^{b^{\prime} 0} C_{s 0 b^{\prime} \beta}^{b \beta},
\end{aligned}
$$

и убедиться в том, что их правые части равны нулю, если не выполнено условие треугольника $\mathbf{b}=\mathbf{b}^{\prime}+\mathbf{s}$ или если $\left(b+s-b^{\prime}\right)$ - нечетное число.

\section{3. СТРОЕНИЕ ОБЩЕГО РЕГУЛЯРНОГО РЕШЕНИЯ}

Докажем, что общее регулярное решение $\Psi$ уравнения Шредингера (2) со взаимодействиями (5) является формальным рядом по целым степеням $x$,

$$
\Psi(\mathbf{x}, \mathbf{y})=\sum_{n=0}^{\infty} x^{n} \Psi^{n}(\hat{x}, \mathbf{y})
$$

а каждая компонента $\Psi^{n}$ этого ряда ортогональна любой сферической гармонике $Y_{b \beta}(\hat{x})$ c $b>n$, т.е. представима, вообше говоря, в виде конечного сферического ряда с ненулевыми сферическими компонентами $\Psi_{b \beta}^{n}, b \leqslant n$,

$$
\Psi^{n}(\hat{x}, \mathbf{y})=\sum_{b=0}^{n} \sum_{\beta=-b}^{b} \Psi_{b \beta}^{n}(\mathbf{y}) Y_{b \beta}(\hat{x}), \quad \Psi_{b \beta}^{n}(\mathbf{y}) \equiv\left\langle Y_{b \beta}(\hat{x}) \mid \Psi(\hat{x}, \mathbf{y})\right\rangle
$$

Учитывая разложение (17), для оператора $H$ можно записать представление

$$
\begin{gathered}
H(\mathbf{x}, \mathbf{y})=-\partial_{x}^{2}-\frac{2}{x} \partial_{x}+\frac{\mathbf{l}_{x}^{2}}{x^{2}}+\frac{q}{x}+\sum_{p=1}^{\infty} x^{p} \sum_{s=0}^{p} V^{p s}(y) P_{s}(u)+h(\mathbf{y})+E \\
h(\mathbf{y}) \equiv-\partial_{y}^{2}-\frac{2}{y} \partial_{y}+\frac{\mathbf{l}_{y}^{2}}{y^{2}}+V^{00}(y)-E, \quad \mathbf{l}_{x} \equiv-i \mathbf{x} \times \nabla_{x}, \quad \mathbf{l}_{y} \equiv-i \mathbf{y} \times \nabla_{y} .
\end{gathered}
$$


Уравнение Шредингера (2) после подстановки (21) сводится к рекуррентной по индексу $n$ цепочке уравнений

$$
\begin{aligned}
& \mathbf{l}_{x}^{2} \Psi^{0}(\hat{x}, \mathbf{y})=0 \\
&\left(\mathbf{l}_{x}^{2}-2\right) \Psi^{1}(\hat{x}, \mathbf{y})=-q \Psi^{0}(\hat{x}, \mathbf{y}), \\
& {\left[\mathbf{l}_{x}^{2}-(n+2)(n+3)\right] \Psi^{n+2}(\hat{x}, \mathbf{y})=-q \Psi^{n+1}(\hat{x}, \mathbf{y})-h(\mathbf{y}) \Psi^{n}(\hat{x}, \mathbf{y})-} \\
&-\sum_{p=1}^{n} \sum_{s=0}^{p} V^{p s}(y) P_{s}(u) \Psi^{n-p}(\hat{x}, \mathbf{y}), \quad n=0,1, \ldots
\end{aligned}
$$

Поэтому доказательство сушествования представления (21) сводится к доказательству разрешимости такой цепочки. Согласно теории дифференциальных уравнений [16] уравнение для $\Psi^{n+2}, n \geqslant-2$, разрешимо тогда и только тогда, когда его правая часть ортогональна обшему решению соответствуюшего однородного уравнения, т.е. всем гармоникам $Y_{n+2, \beta}(\hat{x}) \mathrm{c}|\beta| \leqslant n+2$. Докажем такую ортогональность по индукшии.

Общее регулярное решение уравнения (25) является произведением гармоники $Y_{00}(\hat{x})$ и произвольной функции $\Psi_{00}^{0}$ аргумента $\mathbf{y}$,

$$
\Psi^{0}(\hat{x}, \mathbf{y})=\Psi_{00}^{0}(\mathbf{y}) Y_{00}(\hat{x}) .
$$

Поэтому правая часть уравнения (26) для неизвестной функции $\Psi^{1}$ ортогональна гармоникам $Y_{1 \beta}(\hat{x}), \beta=0, \pm 1$. Следовательно, решение $\Psi^{1}$ сушествует и равно сумме частного решения $q \Psi^{0} / 2$ исследуемого неоднородного уравнения и обшего решения соответствуюшего однородного уравнения, т.е.

$$
\Psi^{1}(\hat{x}, \mathbf{y})=\frac{q}{2} \Psi_{00}^{0}(\mathbf{y}) Y_{00}(\hat{x})+\sum_{\beta=0, \pm 1} \Psi_{1 \beta}^{1}(\mathbf{y}) Y_{1 \beta}(\hat{x}) .
$$

Итак, решения $\Psi^{0}$ и $\Psi^{1}$ - это ряды $(22)$ с произвольными компонентами $\Psi_{00}^{0}$ и $\Psi_{1 \beta}^{1}$. Следовательно, первый этап доказательства по индукции выполнен. Перейдем ко второму этапу. Предположим, что при некотором $n$ все функции $\Psi^{n^{\prime}}$ с $n^{\prime} \leqslant n+1$ представляют собой известные конечные суммы $(22)$, но искомое решение $\Psi^{n+2}$, вообще говоря, является бесконечным сферическим рядом. В уравнении $(27)$ заменим все функции $\Psi^{n^{\prime}}$ с $n^{\prime} \leqslant n+2$ их рядами. С помошью (18) спроецируем получившееся уравнение на сферический базис (6). В итоге для искомых проекций $\Psi_{b \beta}^{n+2}(\mathbf{y})$ получатся алгебраические и не зацепляюшиеся ни по индексу $b=0,1, \ldots$, ни по индексу $\beta=-b, \ldots, b$ уравнения

$$
\begin{aligned}
& {[b(b+1)-(n+2)(n+3)] \Psi_{b \beta}^{n+2}(\mathbf{y})=-q \Psi_{b \beta}^{n+1}(\mathbf{y})-h(\mathbf{y}) \Psi_{b \beta}^{n}(\mathbf{y})-} \\
& \quad-\sum_{p=1}^{n} \sum_{s=0}^{p}(-1)^{s}\left(\frac{4 \pi}{2 s+1}\right)^{1 / 2} V^{p s}(y) \sum_{b^{\prime}=0}^{n-p} \sum_{\beta^{\prime}=-b^{\prime}}^{b^{\prime}} C_{s 0 b 0}^{b^{\prime} 0} C_{s \alpha b \beta^{\prime}}^{b \beta} Y_{s \alpha}^{*}(\hat{y}) \Psi_{b^{\prime} \beta^{\prime}}^{n-p}(\mathbf{y}) .
\end{aligned}
$$

В этих уравнениях $b^{\prime} \leqslant n-p$, а $s \leqslant p$, т.е. $b^{\prime}+s \leqslant n$. Поэтому при $b>n$ все коэффициенты $C_{s 0 b 0}^{b^{\prime} 0}$, а значит, и сумма по индексам $b^{\prime}, \beta^{\prime}$ обрашаются в нуль. Следовательно, при $b>n+2$, когда по предположению индукции $\Psi_{b \beta}^{n+1}, \Psi_{b \beta}^{n}=0$, уравнения (30) становятся однородными и имеют только тривиальные решения $\Psi_{b \beta}^{n+2} \equiv 0,|\beta| \leqslant b$. При $b=n+2$

4 Теоретическая и математическая физика, т. 136, № 1, 2003 г. 
и любом $\beta=-b, \ldots, b$ эти уравнения становятся тождествами типа $0 \Psi_{n+2, \beta}^{n+2}=0$. Им подчиняются произвольные функции $\Psi_{n+2, \beta}^{n+2}(\mathbf{y})$. Так как при $b \leqslant n+1$ исследуемые уравнения (30) всегда имеют нетривиальные решения $\Psi_{b \beta}^{n+2},|\beta| \leqslant b$, то $\Psi^{n+2}$ - конечная сумма типа (22). Следовательно, вся цепочка исходных уравнений (25)-(27) разрешима, а для всех ее решений $\Psi^{n}$ верны представления (22), что и требовалось доказать. Попутно было показано, что все сферические компоненты $\Psi_{b \beta}^{n},|\beta| \leqslant b$, с максимально возможным при данном $n$ значении $b=n$ являются произвольными функциями переменной $\mathbf{y}$, через которые однозначно выражаются все остальные компоненты $\Psi_{b \beta}^{n}$ с $b<n$. Вывод таких представлений несложен и заключается в решении уравнений (30) в порядке возрастания индекса $n$ и убывания индекса $b$ при данном $n$.

Поясним этот вывод примером. Подставим компоненты $\Psi_{b \beta}^{n}, n=0,1$, функций (28) и (29) в правые части уравнений $(30)$ с $n=0$ и найдем решения,

$$
\Psi_{1 \beta}^{2}(\mathbf{y})=\frac{q}{4} \Psi_{1 \beta}^{1}(\mathbf{y}), \quad \Psi_{00}^{2}(\mathbf{y})=\frac{1}{12}\left[2 h(\mathbf{y})+q^{2}\right] \Psi_{00}^{0}(\mathbf{y})
$$

Далее, используя эти выражения, решим уравнения (30) с $n=1$,

$$
\begin{aligned}
& \Psi_{2 \beta}^{3}(\mathbf{y})=\frac{q}{6} \Psi_{2 \beta}^{2}(\mathbf{y}), \\
& \Psi_{1 \beta}^{3}(\mathbf{y})=\frac{1}{40}\left[4 h(\mathbf{y})+q^{2}\right] \Psi_{1 \beta}^{1}(\mathbf{y})+\frac{\sqrt{\pi}}{15} V^{11}(\mathbf{y}) \Psi_{00}^{0}(\mathbf{y}) Y_{1 \beta}^{*}(\hat{y}), \\
& \Psi_{00}^{3}(\mathbf{y})=\frac{1}{144}\left[q\left(8 h(\mathbf{y})+q^{2}\right)+12 V^{10}\right] \Psi_{00}^{0}(\mathbf{y}) .
\end{aligned}
$$

Увеличивая $n$ от $n=2$, можно построить функции $\Psi_{b \beta}^{n+2}$ с $b=n+1, n, \ldots, 0$ :

$$
\begin{gathered}
\Psi_{n+1, \beta}^{n+2}(\mathbf{y})=\frac{q}{2(n+2)} \Psi_{n+1, \beta}^{n+1}(\mathbf{y}), \\
\Psi_{n \beta}^{n+2}(\mathbf{y})=\frac{1}{2(2 n+3)}\left[h(\mathbf{y})+\frac{q^{2}}{2(n+1)}\right] \Psi_{n \beta}^{n}(\mathbf{y})+ \\
+\frac{\sqrt{4 \pi}}{2(2 n+3)} \sum_{p=1}^{n} \frac{(-1)^{p}}{\sqrt{2 p+1}} C_{n 0 p 0}^{n-p, 0} V^{p p}(\mathbf{y}) \sum_{\beta^{\prime}=p-n}^{n-p} C_{p \alpha, n-p, \beta^{\prime}}^{n \beta} \Psi_{n-p, \beta^{\prime}}^{n-p}(\mathbf{y}) Y_{p \alpha}^{*}(\hat{y}), \ldots
\end{gathered}
$$

Отметив, что асимптотика функции $\Psi$, полученная подстановкой $(28)$ и $(29)$ в (21), совпадает с асимптотикой (12), доказанной в работе [14], перейдем к проблеме построения связей (9) для $\Psi$. Каждый ряд $(22)$ для функции $\Psi^{n}(n=0,1, \ldots)$ содержит $n(n+2)$ неизвестных компонент $\Psi_{b \beta}^{n^{\prime}}(\mathbf{y}), b=n^{\prime} \leqslant n,|\beta| \leqslant b$. Поэтому не сушествует конечной линейной комбинации функций $\Psi^{n}$, не содержащей ни одной неизвестной компоненты и равной нулю при всех $\hat{x}$ и $\mathbf{y}$. Так как $\left.\partial_{x}^{n} \Psi\right|_{x=0}=(n !) \Psi^{n}$, то в исследуемом общем случае, когда среди частиц $p_{1}, p_{2}, p_{3}$ нет тождественных, такую линейную комбинацию, т.е. связь (9), нельзя построить и для частных производных функции $\Psi$. Докажем, что такие связи имеются, если $p_{j}$ и $p_{k}$ - тождественные частицы, а именно бозоны с целыми спинами или фермионы с полуцелыми спинами.

Если частицы имеют спины, то любое решение $\widetilde{\Psi}$ трехчастичного уравнения Шредингера в координатно-спиновом пространстве зависит и от спиновых координат частиц. В 
нашем случае в формулах взаимодействий (5) не содержатся спиновые операторы. Поэтому зависимость от спинов частиц описывается спиновой функцией $\chi$, содержашейся в решении $\widetilde{\Psi}$ в качестве множителя, $\widetilde{\Psi}=\chi \Psi$, а пространственная часть $\Psi$ решения $\widetilde{\Psi}$ подчиняется уравнению Шредингера (2) в координатном пространстве.

Пусть $P_{j k}$ - оператор перестановки $p_{j} \leftrightarrow p_{k}$. Тогда $P_{j k} \widetilde{\Psi}^{ \pm}= \pm \widetilde{\Psi}^{ \pm}$, причем знак плюс или минус берется, если $p_{j}$ и $p_{k}-$ бозоны или фермионы, соответственно. Если $p_{j}$ и $p_{k}-$ бозоны, то $P_{j k} \chi=\chi$ и поэтому возможен только один случай: $P_{j k} \Psi=\Psi$. Если $p_{j}$ и $p_{k}$ - фермионы, то возможны два случая: $P_{j k} \chi=\chi, P_{j k} \Psi=-\Psi$ и $P_{j k} \chi=-\chi$, $P_{j k} \Psi=\Psi$. Из этих случаев достаточно исследовать только первый, потому что второй случай, когда функция $\Psi$ четна относительно перестановки двух фермионов, эквивалентен случаю двух тождественных бозонов.

Перестановка частиц $p_{j}$ и $p_{k}$ приводит к замене $\mathbf{x} \rightarrow-\mathbf{x}$, относительно которой гамильтониан $H$ инвариантен. Следовательно, $H P_{j k}-P_{j k} H=0$ и уравнение Шредингера (2) имеет решения $\Psi^{ \pm}$с определенной перестановочной симметрией: $P_{j k} \Psi^{ \pm}= \pm \Psi^{ \pm}$. В этом соотношении, согласно данным выше пояснениям, выбираем знак плюс, если $p_{j}$ и $p_{k}$ - бозоны, и знак минус, если $p_{j}$ и $p_{k}-$ фермионы в состоянии с четной относительно их перестановки спиновой функцией $\chi$, что и подразумевается всюду далее.

Так как [3]

$$
P_{j k} Y_{b \beta}\left(\theta_{x}, \varphi_{x}\right)=Y_{b \beta}\left(\pi-\theta_{x}, \pi+\varphi_{x}\right)=(-1)^{b} Y_{b \beta}\left(\theta_{x}, \varphi_{x}\right),
$$

то $\Psi^{ \pm}=P^{ \pm} \Psi$, где $\Psi$ - исследованное выше обшее решение, а $P^{+}$и $P^{-}$- ортогональные относительно интегрирования по углам $\hat{x}=\left(\theta_{x}, \varphi_{x}\right)$ проекторы,

$$
P^{ \pm}=\frac{1}{2}\left(1 \pm P_{j k}\right)=\frac{1}{2} \sum_{b=0}^{\infty}\left[1 \pm(-1)^{b}\right] \sum_{\beta=-b}^{b}\left|Y_{b \beta}(\hat{x})\right\rangle\left\langle Y_{b \beta}(\hat{x})\right| .
$$

Для построения разложений $\Psi^{+}$или $\Psi^{-}$достаточно подействовать проектором $P^{+}$ или $P^{-}$на ряды $(21),(22)$ и уравнения $(25)-(27)$. В итоге получатся ряды с компонентами $\Psi_{b \beta}$ общего решения $\Psi$ с четным либо нечетным $b$,

$$
\begin{aligned}
\Psi^{ \pm}(\mathbf{x}, \mathbf{y}) & =\sum_{n=0}^{\infty} x^{n} \Psi^{n \pm}(\hat{x}, \mathbf{y}), \\
\Psi^{n+}(\hat{x}, \mathbf{y}) & =\sum_{b=0,2, \ldots}^{n} \sum_{\beta=-b}^{b} \Psi_{b \beta}^{n}(\mathbf{y}) Y_{b \beta}(\hat{x}), \\
\Psi^{n-}(\hat{x}, \mathbf{y}) & =\sum_{b=1,3, \ldots}^{n} \sum_{\beta=-b}^{b} \Psi_{b \beta}^{n}(\mathbf{y}) Y_{b \beta}(\hat{x}),
\end{aligned}
$$

а компоненты $\Psi_{b \beta}^{n}, n \geqslant 2$, с четным (нечетным) $b$ будут удовлетворять системе $(30)$, в которой $b^{\prime}$ будет только четным (нечетным), а $s$ - всегда четным.

Пусть $p_{j}$ и $p_{k}$ - тождественные бозоны. Тогда представление $(34)$ и проекции $P^{+} \Psi^{0}$ и $P^{+} \Psi^{1}$ функций $(28)$ и $(29)$

$$
\left.\Psi^{+}\right|_{x=0}=\Psi^{0+}=\Psi_{00}^{0} Y_{00}(\hat{x}),\left.\quad \partial_{x} \Psi^{+}\right|_{x=0}=\Psi^{1+}=\frac{q}{2} \Psi^{0+}
$$


порождают связь (9) решения $\Psi^{+}$с его первой производной $\partial_{x} \Psi^{+}$при $x=0$,

$$
\left(2 \partial_{x}-q\right) \Psi^{+}(\mathbf{x}, \mathbf{y})=0, \quad x=0, \quad \forall \hat{x}, \mathbf{y}: y>0 .
$$

Проекции $\Psi^{n+}, n=2,3$, функций $\Psi^{n}$ с компонентами (31) и (32) содержат одинаковое число неизвестных функций $\Psi_{2 \beta}^{2}$ в виде суммы $S$ :

$$
\begin{gathered}
\left.\frac{1}{2 !} \partial_{x}^{2} \Psi^{+}\right|_{x=0}=\Psi^{2+}=\frac{1}{12}\left(2 h+q^{2}\right) \Psi^{0+}+S, \quad S \equiv \sum_{\beta=-2}^{2} \Psi_{2 \beta}^{2} Y_{2 \beta}(\hat{x}), \\
\left.\frac{1}{3 !} \partial_{x}^{3} \Psi^{+}\right|_{x=0}=\Psi^{3+}=\frac{1}{144}\left[q\left(8 h+q^{2}\right)+12 V^{10}\right] \Psi^{0+}+\frac{q}{6} S .
\end{gathered}
$$

Поэтому выражение $6 \Psi^{3+}-q \Psi^{2+}$ не содержит $S$ и дает связь (9) при $x=0$,

$$
12 \partial_{x}^{2}\left(2 \partial_{x}-q\right) \Psi^{+}(\mathbf{x}, \mathbf{y})=\left[q\left(4 h(\mathbf{y})-q^{2}\right)+12 V^{10}\right] \Psi^{+}(\mathbf{x}, \mathbf{y}) .
$$

Если $p_{j}$ и $p_{k}-$ фермионы, то ряд (34) решения $\Psi^{-}$содержит функции

$$
\begin{gathered}
\Psi^{0-} \equiv 0, \quad \Psi^{1-}=\sum_{\beta=0, \pm 1} \Psi_{1 \beta}^{1} Y_{1 \beta}(\hat{x}), \quad \Psi^{2-}=\frac{q}{4} \Psi^{1-}, \\
\Psi^{3-}=\frac{1}{40}\left(4 h+q^{2}\right) \Psi^{1-}+S, \quad S \equiv \sum_{\beta=-3}^{3} \Psi_{3 \beta}^{3} Y_{3 \beta}(\hat{x}), \\
\Psi^{4-}=\frac{1}{720}\left[q\left(14 h+q^{2}\right)+40 V^{10}\right] \Psi^{1-}+\frac{q}{8} S .
\end{gathered}
$$

Поэтому при $x=0$ имеются следуюшие связи:

$$
\begin{gathered}
\partial_{x}\left(2 \partial_{x}-q\right) \Psi^{-}(\mathbf{x}, \mathbf{y})=0 \\
12 \partial_{x}^{3}\left(2 \partial_{x}-q\right) \Psi^{-}(\mathbf{x}, \mathbf{y})=\left[q\left(4 h(\mathbf{y})-q^{2}\right)+32 V^{10}\right] \partial_{x} \Psi^{-}(\mathbf{x}, \mathbf{y}) .
\end{gathered}
$$

Вернемся к случаю нетождественных частиц $p_{j}$ и $p_{k}$ и приступим к анализу строения частного решения $\Psi^{\varepsilon}, \varepsilon=(E, \ell, m, \sigma)$, уравнения Шредингера.

\section{4. СТРОЕНИЕ ФИЗИЧЕСКОГО РЕШЕНИЯ \\ В БИСФЕРИЧЕСКОМ БАЗИСЕ}

В базисе (7) оператор $P^{\varepsilon}$, проецируюший $\Psi$ на $\Psi^{\varepsilon}$, является суммой

$$
\begin{aligned}
P^{\varepsilon} & =\frac{1}{2} \sum_{\mathbf{a}+\mathbf{b}=\mathbf{1}}\left[1+\sigma(-1)^{a+b}\right]\left|\mathcal{Y}_{a b}^{\ell m}(\hat{x}, \hat{y})\right\rangle\left\langle\mathcal{Y}_{a b}^{\ell m}(\hat{x}, \hat{y})\right|= \\
& =\sum_{b=\mu(\sigma)}^{\infty} \sum_{a}\left|\mathcal{Y}_{a b}^{\ell m}(\hat{x}, \hat{y})\right\rangle\left\langle\mathcal{Y}_{a b}^{\ell m}(\hat{x}, \hat{y})\right|, \quad \mu(\sigma) \equiv \frac{1}{2}\left[1-(-1)^{\ell} \sigma\right] .
\end{aligned}
$$

Здесь и далее при заданных $b, \ell$ и $\sigma$ индекс $a=b$, если $\ell=0$, а при $\ell>0$ индекс $a$ вычисляется с помошью функций $\mu(\sigma)$ и $\chi(b) \equiv\left[1-(-1)^{b}\right] / 2$ по формулам

$$
a=a(c ; \ell, b, \sigma)=|\ell-b|+2 c+(-1)^{\ell} \sigma \chi(b)+\mu(\sigma),
$$


где

$$
c=0,1, \ldots, c_{\max }=\frac{1}{2}[\ell+b-|\ell-b|]-(-1)^{\ell} \sigma \chi(b)-\mu(\sigma) .
$$

Образом $\Psi^{\varepsilon}=P^{\varepsilon} \Psi$ ряда (21) является степенной ряд

$$
\Psi^{\varepsilon}(\mathbf{x}, \mathbf{y})=\sum_{n=0}^{\infty} x^{n} \Psi^{n \varepsilon}(\hat{x}, \mathbf{y}), \quad \Psi^{n \varepsilon}(\hat{x}, \mathbf{y}) \equiv P^{\varepsilon} \Psi^{n}(\hat{x}, \mathbf{y})
$$

в котором вследствие соотношений $(22)$ и (38) функции $\Psi^{n \varepsilon}$ представляются конечными суммами,

$$
\Psi^{n \varepsilon}(\hat{x}, \mathbf{y})=\sum_{b=\mu(\sigma)}^{n} \sum_{a} \Psi_{a b}^{n \varepsilon}(y) \mathcal{Y}_{a b}^{\ell m}(\hat{x}, \hat{y}), \quad \Psi_{a b}^{n \varepsilon}(y) \equiv\left\langle\mathcal{Y}_{a b}^{\ell m}(\hat{x}, \hat{y}) \mid \Psi^{n}(\hat{x}, \mathbf{y})\right\rangle
$$

а их компоненты выражаются через проекции функций $\Psi_{b \beta}^{n}$ и $\Psi^{n}$,

$$
\Psi_{a b}^{n \varepsilon}(y)=\sum_{\alpha=-a}^{a} C_{a \alpha b \beta}^{\ell m}\left\langle Y_{a \alpha}(\hat{y}) \mid \Psi_{b \beta}^{n}(\mathbf{y})\right\rangle=\sum_{\alpha=-a}^{a} C_{a \alpha b \beta}^{\ell m}\left\langle Y_{a \alpha}(\hat{y}) Y_{b \beta}(\hat{x}) \mid \Psi^{n}(\hat{x}, \mathbf{y})\right\rangle
$$

Этот же образ можно представить как разложение решения $\Psi^{\varepsilon}$ по базису (7),

$$
\begin{aligned}
\Psi^{\varepsilon}(\mathbf{x}, \mathbf{y}) & =\sum_{a b} \Psi_{a b}^{\varepsilon}(x, y) \mathcal{Y}_{a b}^{\ell m}(\hat{x}, \hat{y}), \\
\Psi_{a b}^{\varepsilon}(x, y) & \equiv\left\langle\mathcal{Y}_{a b}^{\ell m}(\hat{x}, \hat{y}) \mid \Psi(\mathbf{x}, \mathbf{y})\right\rangle=\sum_{n=b \geqslant \mu(\sigma)}^{\infty} x^{n} \Psi_{a b}^{n \varepsilon}(y),
\end{aligned}
$$

и тем самым доказать, что бисферические компоненты $\Psi_{a b}^{\varepsilon}$ такого разложения являются степенными рядами с асимптотиками $\Psi_{a b}^{\varepsilon}=O\left(x^{b}\right), b=\mu(\sigma)$, при $x \rightarrow 0$.

Как было показано, функции $\Psi_{b \beta}^{n}$ с $b=n=0,1, \ldots$ нельзя определить, но через них можно выразить все функции $\Psi_{b \beta}^{n} \mathrm{c} b \leqslant n$. Поэтому при $b=n$ и любом $a$ суммы (41) являются произвольными функциями $\Psi_{a n}^{n \varepsilon}$, через которые однозначно представляются все остальные функции $\Psi_{a b}^{n \varepsilon} \mathrm{c} b \leqslant n$. Это утверждение можно доказать и другим способом, применив метод индукции к рекуррентной по индексу $n$ цепочке алгебраических уравнений, полученной проецированием цепочки (25)-(27) с помощью формул (19) и (38),

$$
\begin{gathered}
{[b(b+1)-(n+2)(n+3)] \Psi_{a b}^{n+2, \varepsilon}(y)=-q \Psi_{a b}^{n+1, \varepsilon}(y)-h_{a}(y) \Psi_{a b}^{n \varepsilon}(y)-} \\
-(-1)^{a+b-\ell} \sum_{p=1}^{n} \sum_{s=0}^{p}(-1)^{s} V^{p s}(y) \sum_{b^{\prime}=\mu(\sigma)}^{n-p}\left(2 b^{\prime}+1\right)^{1 / 2} \sum_{a^{\prime}}\left(2 a^{\prime}+1\right)^{1 / 2} \times \\
\times C_{s 0 a^{\prime} 0}^{a 0} C_{s 0 b^{\prime} 0}^{b 0}\left\{\begin{array}{ccc}
a & a^{\prime} & s \\
b^{\prime} & b & \ell
\end{array}\right\} \Psi_{a^{\prime} b^{\prime}}^{n-p, \varepsilon}(y), \\
n=-2,-1, \ldots, \quad b=\mu(\sigma), 1, \ldots, n,
\end{gathered}
$$


где $h_{a}$ - диагональный матричный элемент оператора (24) в базисе $(7)$,

$$
\begin{gathered}
\left\langle\mathcal{Y}_{a b}^{\ell m}|h(\mathbf{y})| \mathcal{Y}_{a^{\prime} b^{\prime}}^{\ell m}\right\rangle=\delta_{a a^{\prime}} \delta_{b b^{\prime}} h_{a}(y), \\
h_{a}(y) \equiv-\partial_{y}^{2}-\frac{2}{y} \partial_{y}+\frac{a(a+1)}{y^{2}}+V^{00}(y)-E .
\end{gathered}
$$

Доказательство разрешимости цепочки (43) принципиально не отличается от данного выше анализа системы (27) и поэтому опускается.

Компоненты $\Psi^{n \varepsilon}$ разложения (39) физического решения $\Psi^{\varepsilon}$ можно найти, если известны компоненты $\Psi^{n}$ или $\Psi_{b \beta}^{n}$ общего решения $\Psi$. Например, подействовав проектором (38) на функции (28) и (29), получаем

$$
\begin{array}{ll}
\Psi^{0 \varepsilon}(\hat{x}, \mathbf{y})=\Psi_{\ell 0}^{0 \varepsilon}(y) \mathcal{Y}_{\ell 0}^{\ell m}(\hat{x}, \hat{y}), & \\
\Psi^{1 \varepsilon}(\hat{x}, \mathbf{y})=\frac{q}{2} \Psi^{0 \varepsilon}(\hat{x}, \mathbf{y})+\sum_{a=|\ell \pm 1|} \Psi_{a 1}^{1 \varepsilon}(y) \mathcal{Y}_{a 1}^{\ell m}(\hat{x}, \hat{y}), & \sigma=(-1)^{\ell}, \\
\Psi^{0 \varepsilon}(\hat{x}, \mathbf{y})=\Psi_{\ell 0}^{0 \varepsilon}(y) \equiv 0, \quad \Psi^{1 \varepsilon}(\hat{x}, \mathbf{y})=\Psi_{\ell 1}^{1 \varepsilon}(y) \mathcal{Y}_{\ell 1}^{\ell m}(\hat{x}, \hat{y}), \quad \sigma=(-1)^{\ell+1},
\end{array}
$$

а подставив сферические компоненты (31) и (32) в суммы (41) и затем упростив ряды (40), доказываем, что при любом $\sigma$

$$
\begin{aligned}
\Psi^{2 \varepsilon}= & \frac{1}{12}\left(2 h_{\ell}+q^{2}\right) \Psi^{0 \varepsilon}+\frac{q}{4} \sum_{a} \Psi_{a 1}^{1 \varepsilon} \mathcal{Y}_{a 1}^{\ell m}+\sum_{a} \Psi_{\ell 2}^{2 \varepsilon} \mathcal{Y}_{a 2}^{\ell m} \\
\Psi^{3 \varepsilon}= & \frac{1}{144}\left[q\left(8 h_{\ell}+q^{2}\right)+12 V^{10}\right] \Psi^{0 \varepsilon}+\frac{1}{40} \sum_{a}\left(4 h_{a}+q^{2}\right) \Psi_{a 1}^{1 \varepsilon} \mathcal{Y}_{a 1}^{\ell m}- \\
& -\frac{V^{11}}{10 \sqrt{3}} \Psi_{\ell 0}^{0 \varepsilon} \sum_{a=|\ell \pm 1|} C_{\ell 010}^{a 0} \mathcal{Y}_{a 1}^{\ell m}+\frac{q}{6} \sum_{a} \Psi_{a 2}^{2 \varepsilon} \mathcal{Y}_{a 2}^{\ell m}+\sum_{a} \Psi_{a 3}^{3 \varepsilon} \mathcal{Y}_{a 3}^{\ell m} .
\end{aligned}
$$

Третий способ построения функций $\Psi^{n \varepsilon}$ реализуется подстановкой решений $\Psi_{a b}^{n \varepsilon}$ системы (43) в суммы (40). При этом компоненты $\Psi^{n}$ или $\Psi_{b \beta}^{n}$ не требуются. Поэтому такой способ представляется оптимальным для вывода как асимптотики ряда (39), так и асимптотик компонент $\Psi_{a b}^{\varepsilon}$ разложения (42).

Выразив решения $\Psi_{a b}^{n \varepsilon}, n \leqslant 4$, цепочки (43) через неизвестные функции $\left.\partial_{x}^{n} \Psi_{a b}^{\varepsilon}\right|_{x=0}=$ $(n !) \Psi_{a b}^{n \varepsilon}$ с $b=n$, получаем следуюшие асимптотические формулы:

$$
\begin{aligned}
\Psi_{\ell 0}^{\varepsilon}(x, y)= & \left\{1+\frac{q x}{2}+\frac{x^{2}}{12}\left[2 h_{\ell}(y)+q^{2}\right]+\frac{x^{3}}{144}\left[q\left(8 h_{\ell}(y)+q^{2}\right)+12 V^{10}\right]+\right. \\
& \left.+\frac{x^{4}}{20}\left[\frac{1}{36} h_{\ell}(y)\left[6 h_{\ell}(y)+5 q^{2}\right]+\frac{q^{4}}{144}+V^{20}(y)+\frac{7 q}{12} V^{10}\right]\right\} \Psi_{\ell 0}^{\varepsilon}(0, y)- \\
& -\left.\frac{\sqrt{3}}{60} x^{4} V^{11}(y) \sum_{a^{\prime}=|\ell \pm 1|} C_{\ell 010}^{a^{\prime} 0} \partial_{x} \Psi_{a^{\prime} 1}^{\varepsilon}(x, y)\right|_{x=0}+O\left(x^{5}\right) \\
\Psi_{a 1}^{\varepsilon}(x, y)= & \left\{1+\frac{q x}{4}+\frac{x^{2}}{40}\left[4 h_{\ell}(y)+q^{2}\right]+\right. \\
& \left.+\frac{x^{3}}{720}\left[q\left(14 h_{\ell}(y)+q^{2}\right)+40 V^{10}\right]\right\}\left.\partial_{x} \Psi_{a 1}^{\varepsilon}(x, y)\right|_{x=0}- \\
& -\frac{\sqrt{3}}{270} V^{11}(y) C_{\ell 010}^{a 0}(9+2 q x) x^{3} \Psi_{\ell 0}^{\varepsilon}(0, y)+O\left(x^{5}\right)
\end{aligned}
$$


где $a=|\ell \pm(1-\mu(\sigma))|$,

$$
\begin{aligned}
\Psi_{a 2}^{\varepsilon}(x, y)= & \left.\frac{x^{2}}{2}\left\{1+\frac{q x}{6}+\frac{x^{2}}{84}\left[6 h_{\ell}(y)+q^{2}\right]\right\} \partial_{x}^{2} \Psi_{a 2}^{\varepsilon}(x, y)\right|_{x=0}-(-1)^{\ell+a} \frac{x^{4}}{7} V^{11}(y) \times \\
& \times\left.\sum_{a^{\prime}=|\ell \pm 1|}\left(2 a^{\prime}+1\right)^{1 / 2} C_{a^{\prime} 010}^{a 0}\left\{\begin{array}{ccc}
a & a^{\prime} & 1 \\
1 & 2 & 1
\end{array}\right\} \partial_{x} \Psi_{a^{\prime} 1}^{\varepsilon}(x, y)\right|_{x=0}+ \\
& +\frac{x^{4}}{14 \sqrt{5}} V^{22}(y) C_{\ell 020}^{a 0} \Psi_{\ell 0}^{\varepsilon}(0, y)+O\left(x^{5}\right),
\end{aligned}
$$

где $a=|\ell \pm \mu(\sigma)|,|\ell \pm(2-\mu(\sigma))|$.

Вычисляя частные производные $\partial_{x}^{n}, n \leqslant 4$, от этих асимптотик, получаем следуюшие связи (9) при $x=0$ и $y>0$. Для компоненты $\Psi_{\ell 0}^{\varepsilon}$ имеем

$$
\begin{gathered}
\left(2 \partial_{x}-q\right) \Psi_{\ell 0}^{\varepsilon}=0, \quad\left(6 \partial_{x}^{2}-2 h_{\ell}-q^{2}\right) \Psi_{\ell 0}^{\varepsilon}=0, \\
{\left[24 \partial_{x}^{3}-q\left(8 h_{\ell}+q^{2}\right)-12 V^{10}\right] \Psi_{\ell 0}^{\varepsilon}=0,} \\
{\left[120\left(6 V^{20}-\partial_{x}^{4}\right)+5 h_{\ell}\left(6 h_{\ell}+5 q^{2}\right)+q^{4}+84 q V^{10}\right] \Psi_{\ell 0}^{\varepsilon}=} \\
=48 \sqrt{3} V^{11} \sum_{a^{\prime}=|\ell \pm 1|} C_{\ell 010}^{a^{\prime} 0} \partial_{x} \Psi_{a^{\prime} 1}^{\varepsilon} .
\end{gathered}
$$

Для компонент $\Psi_{a 1}^{\varepsilon}, a=|\ell \pm(1-\mu(\sigma))|$, имеем

$$
\begin{aligned}
\partial_{x}\left(2 \partial_{x}-q\right) \Psi_{a 1}^{\varepsilon} & =0 \\
{\left[20 \partial_{x}^{3}-3\left(4 h_{a}+q^{2}\right) \partial_{x}\right] \Psi_{a 1}^{\varepsilon} } & =-4 \sqrt{3} V^{11} C_{\ell 010}^{a 0} \Psi_{\ell 0}^{\varepsilon}, \\
\left\{30 \partial_{x}^{4}-\left[q\left(14 h_{a}+q^{2}\right)+40 V^{10}\right] \partial_{x}\right\} \Psi_{a 1}^{\varepsilon} & =-\frac{16}{\sqrt{3}} q V^{11} C_{\ell 010}^{a 0} \Psi_{\ell 0}^{\varepsilon},
\end{aligned}
$$

а для компонент $\Psi_{a 2}^{\varepsilon}, a=|\ell \pm \mu(\sigma)|,|\ell \pm(2-\mu(\sigma))|$, находим

$$
\begin{aligned}
\partial_{x}^{2}\left(2 \partial_{x}-q\right) \Psi_{a 2}^{\varepsilon}=0 & \\
7 \sqrt{5} \partial_{x}^{4} \Psi_{a 2}^{\varepsilon}= & 12 V^{22} C_{\ell 020}^{a 0} \Psi_{\ell 0}^{\varepsilon}- \\
& -24 \sqrt{5}(-1)^{\ell+a} \sum_{a^{\prime}=|a \pm 1|}\left(2 a^{\prime}+1\right)^{1 / 2} C_{a^{\prime} 010}^{a 0}\left\{\begin{array}{lll}
a & a^{\prime} & 1 \\
1 & 2 & 1
\end{array}\right\} \partial_{x} \Psi_{a^{\prime} 1}^{\varepsilon} .
\end{aligned}
$$

Чтобы воспроизвести соотношения (10) и (11), рассмотрим асимптотику $\Psi^{\varepsilon} \sim \Psi^{\varepsilon 0}+$ $x \Psi^{\varepsilon 1}$ ряда (39) с компонентами (45) в частном случае $\ell=0, m_{3}=\infty, \mathbf{x} \equiv \mathbf{x}_{2}$, когда согласно (1) и (4) $\mathbf{x}=\mathbf{a}_{1} \sqrt{2 m_{1}}$ и $q=z_{3} \sqrt{2 m_{1}}$. Представим второе слагаемое суммы $\Psi^{1 \varepsilon}$ (см. (45)) скалярным произведением $\left(\mathbf{a}_{1} \mathbf{A}\right)$ с неопределенным вектором $\mathbf{A}$,

$$
\Psi^{1 \varepsilon}(y) \mathcal{Y}_{11}^{00}(\hat{x}, \hat{y})=-\frac{\sqrt{3}}{4 \pi} \Psi_{11}^{1 \varepsilon}(y) u=\left(\mathbf{a}_{1} \mathbf{A}\right), \quad \mathbf{A} \equiv-\frac{\sqrt{3}}{4 \pi} \Psi_{11}^{1 \varepsilon}(y) \frac{\mathbf{y}}{a_{1} y} .
$$

Тогда исследуемая асимптотика сведется к равенству (11), доказанному в [13], а после ее интегрирования по всем углам $\hat{x}, \hat{y}$ получится условие Като [12] в его исходной форме (10). Этим замечанием закончим анализ решения $\Psi^{\varepsilon}$ в бисферическом базисе и перейдем к исследованию этого же решения, но в $D$-базисе (8). 


\section{5. СТРОЕНИЕ ФИЗИЧЕСКОГО РЕШЕНИЯ В $D$-БАЗИСЕ}

Введем две "подвижные" системы координат $S^{t}=\left(\mathbf{e}_{1}^{t}, \mathbf{e}_{2}^{t}, \mathbf{e}_{3}^{t}\right), t=x, y$. Пусть начальные точки $O^{x}$ и $O^{y}$ систем $S^{x}$ и $S^{y}$ совпадают с начальной точкой $O$ фиксированной системы $S=\left(\mathbf{e}_{1}, \mathbf{e}_{2}, \mathbf{e}_{3}\right)$, а их реперы таковы, что

$$
\mathbf{y} \mathbf{e}_{1}^{x}>0, \quad \mathbf{y} \mathbf{e}_{2}^{x}=0, \quad \mathbf{x} \mathbf{e}_{3}^{x}=x, \quad \mathbf{x} \mathbf{e}_{1}^{y}<0, \quad \mathbf{x e}_{2}^{y}=0, \quad \mathbf{y} \mathbf{e}_{3}^{y}=y .
$$

Тогда орты $\mathbf{e}_{2}^{x}$ и $\mathbf{e}_{2}^{y}$ направлены вдоль нормали $\mathbf{n}=\mathbf{x} \times \mathbf{y}$ к плоскости трех частиц, система $S^{x}$ ориентирована так же, как и система $S^{R}$ в работах [6], [7], и получается поворотом системы $S^{y}$ вокруг орта $\mathbf{e}_{2}^{y}$ на угол $\theta$ между векторами $\mathbf{x}$ и $\mathbf{y}$. Так как в системе $S$ ориентация этих векторов задана углами $\hat{x}=\left(\theta_{x}, \varphi_{x}\right)$ и $\hat{y}=\left(\theta_{y}, \varphi_{y}\right)$, а в системе $S^{t}$ - углами $\hat{x}^{t}$ и $\hat{y}^{t}$,

$$
\begin{array}{ll}
\hat{x}^{t}=\left(\theta_{x}^{x}, \varphi_{x}^{x}\right)=(0,0), & \hat{y}^{t}=\left(\theta_{y}^{x}, \varphi_{y}^{x}\right)=(\theta, 0), \quad t=x, \\
\hat{x}^{t}=\left(\theta_{x}^{y}, \varphi_{x}^{y}\right)=(\theta, \pi), & \hat{y}^{t}=\left(\theta_{y}^{y}, \varphi_{y}^{y}\right)=(0,0), \quad t=y,
\end{array}
$$

то переход $S \rightarrow S^{x}$ определяется углами Эйлера $\omega^{x}=\left(\varphi_{x}, \theta_{x}, \gamma^{x}\right)$, а переход $S \rightarrow S^{y}-$ углами Эйлера $\omega^{y}=\left(\varphi_{y}, \theta_{y}, \gamma^{y}\right)$, где углы $\gamma^{t}$ таковы, что

$$
\cos \gamma^{t}=\operatorname{ctg} \theta \cos \theta_{t}-\operatorname{cosec} \theta \cos \theta_{t^{\prime}}, \quad t \neq t^{\prime}=x, y .
$$

В $D$-базисе (8) оператор, проецируюший функцию $\Psi$ на $\Psi^{\varepsilon}$, имеет вид

$$
P^{\varepsilon t}=\sum_{m^{\prime}=\mu(\sigma)}^{\ell}\left|D_{m m^{\prime}}^{\ell \sigma *}\left(\omega^{t}\right)\right\rangle\left\langle D_{m m^{\prime}}^{\ell \sigma *}\left(\omega^{t}\right)\right|, \quad t=x, y .
$$

Для построения $D$-компонент $\Psi_{m^{\prime}}^{\varepsilon t}$ образа $\Psi^{\varepsilon}=P^{\varepsilon t} \Psi$,

$$
\Psi^{\varepsilon}(\mathbf{x}, \mathbf{y})=\sum_{m^{\prime}=\mu(\sigma)}^{\ell} \Psi_{m^{\prime}}^{\varepsilon t}(x, y, \theta) D_{m m^{\prime}}^{\ell \sigma *}\left(\omega^{t}\right)
$$

как проекций

$$
\Psi_{m^{\prime}}^{\varepsilon t}(x, y, \theta) \equiv\left\langle D_{m m^{\prime}}^{\ell \sigma *}\left(\omega^{t}\right) \mid \Psi(\mathbf{x}, \mathbf{y})\right\rangle
$$

сферического и бисферического рядов $(21),(22)$ и (40) решения $\Psi$, потребуются разложения функций $Y_{a \alpha}(\hat{y}) Y_{b \beta}(\hat{x})$ и $\mathcal{Y}_{a b}^{\ell m}(\hat{x}, \hat{y})$ по функциям $D_{m m^{\prime}}^{\ell \sigma *}$. Выведем такие разложения.

Используем равенства (6), формулы (53), известные [3] правила преобразования гармоник $Y_{f e}(\hat{a})$ при переходе $S \rightarrow S^{t}$, их значения при нулевых аргументах,

$$
Y_{f e}(\hat{a})=\sum_{e^{\prime}=-f}^{f} D_{e e^{\prime}}^{\ell *}\left(\omega^{t}\right) Y_{f e^{\prime}}\left(\hat{a}^{t}\right), \quad Y_{f e}(0,0)=\left(\frac{2 f+1}{4 \pi}\right)^{1 / 2} \delta_{e 0}, \quad a, t=x, y,
$$


и разложение произведения двух $D$-функций по функциям $D_{m m^{\prime}}^{\ell \sigma *}$ Тогда получим первое искомое разложение

$$
\begin{gathered}
Y_{a \alpha}(\hat{y}) Y_{b \beta}(\hat{x})=\sum_{m^{\prime}=\mu(\sigma)}^{\ell}\left\langle D_{m m^{\prime}}^{\ell \sigma *}\left(\omega^{t}\right) \mid Y_{a \alpha}(\hat{y}) Y_{b \beta}(\hat{x})\right\rangle D_{m m^{\prime}}^{\ell \sigma *}\left(\omega^{t}\right), \\
\left\langle D_{m m^{\prime}}^{\ell \sigma *}\left(\omega^{t}\right) \mid Y_{a \alpha}(\hat{y}) Y_{b \beta}(\hat{x})\right\rangle=\left[\frac{1+\sigma(-1)^{a+b}}{1+\delta_{m^{\prime} 0}}\right]^{1 / 2}(-1)^{m+m^{\prime} \times} \\
\times C_{a \alpha b \beta}^{\ell-m}\left\{\begin{array}{l}
(-1)^{a} C_{a-m^{\prime} \ell m^{\prime}}^{b 0} \Theta_{a m^{\prime}}(u), \\
(-1)^{b+m^{\prime}} C_{b-m^{\prime} \ell m^{\prime}}^{a 0} \Theta_{b m^{\prime}}(u), \quad t=x,
\end{array}\right.
\end{gathered}
$$

Для вывода второго разложения умножим каждое $(\alpha=-a, \ldots a ; \beta=m-a)$ равенство (56) на соответствуюший коэффициент $C_{a \alpha b \beta}^{\ell m}$ и просуммируем полученные равенства по всем $\alpha$. Тогда вследствие определения (7) и свойства ортогональности коэффициентов Клебша-Гордана при $t=x$ получим известное разложение [17], а в случае $t=y$ его аналог

$$
\begin{gathered}
\mathcal{Y}_{a b}^{\ell m}(\hat{x}, \hat{y})=\sum_{m^{\prime}=\mu(\sigma)}^{\ell}\left\langle D_{m m^{\prime}}^{\ell \sigma *}\left(\omega^{t}\right) \mid \mathcal{Y}_{a b}(\hat{x}, \hat{y})\right\rangle D_{m m^{\prime}}^{\ell \sigma *}\left(\omega^{t}\right), \\
\left\langle D_{m m^{\prime}}^{\ell \sigma *}\left(\omega^{t}\right) \mid \mathcal{Y}_{a b}(\hat{x}, \hat{y})\right\rangle= \begin{cases}T_{a b}^{\ell m^{\prime}} \Theta_{a m^{\prime}}(u), & t=x, \\
(-1)^{m^{\prime}} T_{b a}^{\ell m^{\prime}} \Theta_{b m^{\prime}}(u), & t=y, \\
T_{a b}^{\ell m^{\prime}} \equiv\left[\frac{1+\sigma(-1)^{a+b}}{1+\delta_{m^{\prime} 0}}\right]^{1 / 2}(-1)^{a+m^{\prime}} C_{a-m^{\prime} \ell m^{\prime}}^{b 0} .\end{cases}
\end{gathered}
$$

Приступим к анализу $D$-компонент $\Psi_{m^{\prime}}^{\varepsilon t}$ ряда (55). Применяя (54) и (56), построим образ $\Psi^{\varepsilon}=P^{\varepsilon t} \Psi$ ряда (21) с компонентами (22) и таким образом докажем, что $D$-компоненты $\Psi_{m^{\prime}}^{\varepsilon t}$ являются рядами по целым степеням $x$ и конечным суммам $\Psi_{m^{\prime}}^{n \varepsilon t}$ проекций $\left\langle Y_{a \alpha} \mid \Psi_{b \beta}^{n}\right\rangle$ компонент $\Psi_{b \beta}^{n}$ обшего решения,

$$
\begin{aligned}
& \Psi_{m^{\prime}}^{\varepsilon t}(x, y, \theta)=\sum_{n=\mu(\sigma)}^{\infty} x^{n} \Psi_{m^{\prime}}^{n \varepsilon t}(y, \theta), \quad m^{\prime}=\mu(\sigma), \ldots, \ell, \quad t=x, y, \\
& \Psi_{m^{\prime}}^{n \varepsilon t}(y, \theta)=\sum_{b=\mu(\sigma)}^{n} \sum_{a}\left\langle D_{m m^{\prime}}^{\ell \sigma *}\left(\omega^{t}\right) \mid Y_{a \alpha}(\hat{y}) Y_{b \beta}(\hat{x})\right\rangle\left\langle Y_{a \alpha}(\hat{y}) \mid \Psi_{b \beta}^{n}(\mathbf{y})\right\rangle .
\end{aligned}
$$

Следовательно, асимптотики $D$-компонент $\Psi_{m^{\prime}}^{\varepsilon t}$, т.е. конечные подсуммы $(n=\mu(\sigma), \ldots$ $\left.\ldots, n^{\prime}<\infty\right)$ рядов $(58)$, можно найти, заменив функции $\Psi_{b \beta}^{n}$ в суммах (59) их явными выражениями, например, при $n \leqslant 3$ - правыми частями равенств (28), (29), (31) и (32).

Другой вывод представления (58) реализуем проецированием бисферического ряда (40) с помошью формул (54) и (57) на $D$-базис (8). В итоге при $t=x$ функция $\Psi_{m^{\prime}}^{n \varepsilon t}$ представляется конечной суммой по индексу $a$,

$$
\Psi_{m^{\prime}}^{n \varepsilon x}(y, \theta)=\sum_{a=m^{\prime}}^{\ell+n-\mu(\sigma)} \Psi_{a m^{\prime}}^{n \varepsilon x}(y) \Theta_{a m^{\prime}}(u),
$$


с $\Theta$-компонентами

$$
\Psi_{a m^{\prime}}^{n \varepsilon x}(y) \equiv\left\langle\Theta_{a m^{\prime}}(u) \mid \Psi_{m^{\prime}}^{n \varepsilon x}(y, \theta)\right\rangle=\sum_{b=m^{\prime}}^{n} T_{a b}^{\ell m^{\prime}} \Psi_{a b}^{n \varepsilon}(y),
$$

а при $t=y-$ конечной суммой по индексу $b$,

$$
\Psi_{m^{\prime}}^{n \varepsilon y}(y, \theta)=\sum_{b=m^{\prime}}^{n} \Psi_{b m^{\prime}}^{n \varepsilon y}(y) \Theta_{b m^{\prime}}(u)
$$

с $\Theta$-компонентами

$$
\Psi_{b m^{\prime}}^{n \varepsilon y}(y) \equiv\left\langle\Theta_{b m^{\prime}}(u) \mid \Psi_{m^{\prime}}^{n \varepsilon y}(\theta, y)\right\rangle=\sum_{a=m^{\prime}}^{\ell+n-\mu(\sigma)}(-1)^{m^{\prime}} T_{b a}^{\ell m^{\prime}} \Psi_{a b}^{n \varepsilon}(y) .
$$

В этих суммах $a \leqslant \ell+n-\mu(\sigma)$, поскольку $\mathbf{a}=\mathbf{l}-\mathbf{b},(-1)^{a+b}=\sigma$ и $b \leqslant n$. Имеем также $a \geqslant m^{\prime}$ и $b \geqslant m^{\prime}$, потому что согласно (57) $T_{a b}^{\ell m^{\prime}}, T_{b a}^{\ell m^{\prime}}=0$ при $a<m^{\prime}$ или $b<m^{\prime}$. По той же причине $\Psi_{a m^{\prime}}^{n \varepsilon x} \equiv 0$ при $m^{\prime}>a$ и $\Psi_{b m^{\prime}}^{n \varepsilon y} \equiv 0$ при $m^{\prime}>b$. Так как $n \geqslant b \geqslant m^{\prime}$, то $\Psi_{b m^{\prime}}^{n \varepsilon y} \equiv 0$, если $m^{\prime}>n$, но, вообше говоря, $\Psi_{a m^{\prime}}^{n \varepsilon x} \neq 0$ при $m^{\prime}>n$.

В отличие от системы функций $\Theta_{c m^{\prime}}(u), m^{\prime}=-c, \ldots, c$, система функций $\Theta_{c m^{\prime}}(u)$, $c=m^{\prime}, m^{\prime}+1, \ldots$, ортонормирована на отрезке $u \in[-1,1]$,

$$
\left\langle\Theta_{c m^{\prime}}(u) \mid \Theta_{c m^{\prime \prime}}(u)\right\rangle \neq \delta_{m^{\prime} m^{\prime \prime}}, \quad\left\langle\Theta_{c m^{\prime}}(u) \mid \Theta_{c^{\prime} m^{\prime}}(u)\right\rangle=\delta_{c c^{\prime}}
$$

Следовательно, формулы (60) и (62) означают, что компоненты $\Psi_{m^{\prime}}^{n \varepsilon x}$ и $\Psi_{m^{\prime}}^{n \varepsilon y}$ могут быть разложены в конечные суммы по ортонормированным системам функций $\Theta_{a m^{\prime}}(u)$ и $\Theta_{b m^{\prime}}(u)$ с индексами $a, b \geqslant m^{\prime}$. Благодаря конечности таких разложений асимптотики рядов (58), т.е. $D$-компонент $\Psi_{m^{\prime}}^{\varepsilon t}$, можно построить заменой в (61) или (63) бисферических компонент $\Psi_{a b}^{n \varepsilon}$ их явными выражениями (например, (45) и (46)) через неопределенные бисферические компоненты $\Psi_{a b}^{n^{\prime} \varepsilon}, b=n^{\prime} \leqslant n$. Согласно (61) и (63) при такой замене функции $\Psi_{m^{\prime}}^{n \varepsilon x}$ и $\Psi_{m^{\prime}}^{n \varepsilon y}$ с данным $n$ выразятся через произвольные функции $f_{a m^{\prime}}^{n^{\prime} x}$ и $f_{m^{\prime}}^{n^{\prime} y}$,

$$
\begin{gathered}
f_{a m^{\prime}}^{n^{\prime} x}(y)=T_{a b}^{\ell m^{\prime}} f_{a}^{n^{\prime} x}(y), \quad f_{a}^{n^{\prime} x}(y) \equiv \Psi_{a b}^{n^{\prime} \varepsilon}(y), \\
f_{m^{\prime}}^{n^{\prime} y}(y)=\sum_{a=m^{\prime}}^{\ell+n-\mu(\sigma)}(-1)^{m^{\prime}} T_{b a}^{\ell m^{\prime}} \Psi_{a b}^{n^{\prime} \varepsilon}(y), \quad b=n^{\prime} \leqslant n .
\end{gathered}
$$

Для полноты приведем равенства, связывающие все введенные компоненты друг с другом. Из (55) и определения систем $S^{x}$ и $S^{y}$ следует, что

$$
\Psi_{m^{\prime}}^{\varepsilon x}(x, y, \theta)=\sum_{m^{\prime \prime}=\mu(\sigma)}^{\ell} D_{m^{\prime} m^{\prime \prime}}^{\ell \sigma *}(0, \theta, 0) \Psi_{m^{\prime \prime}}^{\varepsilon y}(x, y, \theta), \quad m^{\prime}=\mu(\sigma), \ldots, \ell
$$

Поэтому компоненты $\Psi_{m^{\prime}}^{n \varepsilon x}$ и $\Psi_{m^{\prime}}^{n \varepsilon y}$ рядов (58) связаны аналогичным образом,

$$
\Psi_{m^{\prime}}^{n \varepsilon x}(y, \theta)=\sum_{m^{\prime \prime}=\mu(\sigma)}^{\ell} D_{m^{\prime} m^{\prime \prime}}^{\ell \sigma *}(0, \theta, 0) \Psi_{m^{\prime \prime}}^{n \varepsilon y}(y, \theta), \quad m^{\prime}=\mu(\sigma), \ldots, \ell .
$$


Умножим каждый ряд (61) или $(63)\left(m^{\prime}=\mu(\sigma), \ldots, \ell\right)$ на $T_{a b^{\prime}}^{\ell m^{\prime}} /\left(2-\delta_{m^{\prime} 0}\right)$ или на $T_{b a^{\prime}}^{\ell m^{\prime}} /\left(2-\delta_{m^{\prime} 0}\right)$. Суммируя получившиеся равенства по всем $m^{\prime}$, имеем

$$
\begin{aligned}
\Psi_{a b}^{n \varepsilon}(y) & =2 \sum_{m^{\prime}=\mu(\sigma)}^{\min (a, \ell)} \frac{(-1)^{a+m^{\prime}}}{\left(2-\delta_{\left.m^{\prime} 0\right)^{1 / 2}}\right.} C_{a-m^{\prime} \ell m^{\prime}}^{b 0} \Psi_{a m^{\prime}}^{n \varepsilon x}(y)= \\
& =2 \sum_{m^{\prime}=\mu(\sigma)}^{\min (b, \ell)} \frac{(-1)^{b}}{\left(2-\delta_{m^{\prime} 0}\right)^{1 / 2}} C_{b-m^{\prime} \ell m^{\prime}}^{a 0} \Psi_{b m^{\prime}}^{n \varepsilon y}(y) .
\end{aligned}
$$

Просуммировав по всем $b=\mu(\sigma), \ldots, n$ равенства $(67)$, умноженные на соответствуюшие коэффициенты $C_{a-m^{\prime \prime} \ell m^{\prime \prime}}^{b 0}$ получаем, что при $(-1)^{a+b}=\sigma$

$$
\Psi_{a m^{\prime \prime}}^{n \varepsilon x}(y)=\sigma(-1)^{m^{\prime \prime}} \sum_{b=m^{\prime}}^{n} C_{a-m^{\prime \prime} \ell m^{\prime \prime}}^{b 0} \sum_{m^{\prime}=\mu(\sigma)}^{\min (b, \ell)} \sqrt{\frac{2-\delta_{m^{\prime \prime} 0}}{2-\delta_{m^{\prime} 0}}} C_{b-m^{\prime} \ell m^{\prime}}^{a 0} \Psi_{b m^{\prime}}^{n \varepsilon y}(y)
$$

Третий способ построения асимптотик компонент $\Psi_{m^{\prime}}^{\varepsilon t}$ суммы (55) опишем более подробно. Этот способ представляется оптимальным, потому что в нем, в отличие от двух упомянутых выше способов, не нужны ни функции $\Psi_{b \beta}^{n}$, ни функции $\Psi_{a b}^{n \varepsilon}$, а ключевыми являются доказанные представления (58), (60) и (62). Благодаря им для решения уравнения (55) справедливы следуюшие два разложения:

$$
\begin{aligned}
& \Psi^{\varepsilon}(\mathbf{x}, \mathbf{y})=\sum_{n=\mu(\sigma)}^{\infty} x^{n} \sum_{m^{\prime}=\mu(\sigma)}^{\ell} D_{m m^{\prime}}^{\ell \sigma *}\left(\omega^{x}\right) \sum_{a=m^{\prime}}^{\ell+n-\mu(\sigma)} \Psi_{a m^{\prime}}^{n \varepsilon x}(y) \Theta_{a m^{\prime}}(u), \\
& \Psi^{\varepsilon}(\mathbf{x}, \mathbf{y})=\sum_{n=\mu(\sigma)}^{\infty} x^{n} \sum_{m^{\prime}=\mu(\sigma)}^{\ell} D_{m m^{\prime}}^{\ell \sigma *}\left(\omega^{y}\right) \sum_{b=m^{\prime}}^{n} \Psi_{b m^{\prime}}^{n \varepsilon y}(y) \Theta_{b m^{\prime}}(u)
\end{aligned}
$$

по трем системам линейно независимых функций

$$
\left(x^{0}, x^{1}, \ldots\right), \quad\left(D_{m m^{\prime}}^{\ell \sigma *}, m^{\prime}=\mu(\sigma), \ldots, \ell\right) \text { и }\left(\Theta_{a m^{\prime}}, a \geqslant m^{\prime}\right) \text { или }\left(\Theta_{b m^{\prime}}, b \geqslant m^{\prime}\right) .
$$

Поэтому неизвестные функции $\Psi_{a m^{\prime}}^{n \varepsilon t}(y)$ удается подчинить линейным системам уравнений. Для сравнения выведем их в двух случаях: $t=x$ и $t=y$.

Пусть $t=x$, т.е. используется система $S^{x}$. Тогда действие проектора (54) с $t=x$ на цепочку $(25)-(27)$ дает рекуррентную по индексу $n$ цепочку систем уравнений для искомых компонент $\Psi_{m^{\prime}}^{n \varepsilon x}$ ряда (58),

$$
\begin{aligned}
& {\left[\ell(\ell+1)-2 m^{\prime 2}+Q_{m^{\prime} m^{\prime}}(\theta)-(n+2)(n+3)\right] \Psi_{m^{\prime}}^{n+2, \varepsilon x}(y, \theta)-} \\
& \quad-\sum_{m^{\prime \prime}=m^{\prime} \pm 1} \gamma_{m^{\prime} m^{\prime \prime}}^{\ell \sigma} Q_{m^{\prime} m^{\prime \prime}}(\theta) \Psi_{m^{\prime \prime}}^{n+2, \varepsilon x}(y, \theta)= \\
& =-q \Psi_{m^{\prime}}^{n+1, \varepsilon x}(y, \theta)-h_{m^{\prime}}^{x}(y, \theta) \Psi_{m^{\prime}}^{n \varepsilon x}(y, \theta)-\sum_{p=1}^{n} \sum_{s=0}^{p} V^{p s}(y) P_{s}(u) \Psi_{m^{\prime}}^{n-p, \varepsilon x}(y, \theta)
\end{aligned}
$$


Здесь $\Psi_{m^{\prime}}^{n \varepsilon x} \equiv 0$ при $n<0, m^{\prime}=\mu(\sigma), \ldots, \ell$ при каждом $n=-2,1, \ldots$,

$$
\begin{aligned}
\gamma_{m^{\prime}, m^{\prime}+1}^{\ell \sigma} & \equiv\left\{\left[1+\delta_{m^{\prime} 0} \sigma(-1)^{\ell}\right]\left[\ell(\ell+1)-m^{\prime}\left(m^{\prime}+1\right)\right]\right\}^{1 / 2}, \\
\gamma_{m^{\prime}, m^{\prime}-1}^{\ell \sigma} & \equiv\left(1-\delta_{m^{\prime} 0}\right)\left\{\left[1+\delta_{m^{\prime} 1} \sigma(-1)^{\ell}\right]\left[\ell(\ell+1)-m^{\prime}\left(m^{\prime}-1\right)\right]\right\}^{1 / 2} .
\end{aligned}
$$

Оператор $h_{m^{\prime}}^{x}(y, \theta)$ порожден оператором $(24)$,

$h_{m^{\prime}}^{x}(y, \theta) \equiv\left\langle D_{m m^{\prime}}^{\ell \sigma *}\left(\omega^{x}\right)|h(\mathbf{y})| D_{m m^{\prime}}^{\ell \sigma *}\left(\omega^{x}\right)\right\rangle=-y^{-2} \partial_{y}\left(y^{2} \partial_{y}\right)+y^{-2} Q_{m^{\prime} m^{\prime}}(\theta)+V^{00}(y)-E$,

а операторы $Q_{m^{\prime} m^{\prime \prime}}(\theta)$ определены как

$$
Q_{m^{\prime} m^{\prime}}(\theta) \equiv-\frac{1}{\sin \theta} \partial_{\theta}\left(\sin \theta \partial_{\theta}\right)+\frac{m^{\prime 2}}{\sin ^{2} \theta}, \quad Q_{m^{\prime}, m^{\prime} \pm 1}(\theta) \equiv \mp \partial_{\theta}+\left(m^{\prime} \mp 1\right) \operatorname{ctg} \theta .
$$

При действии на функцию $\Theta_{a m^{\prime}}$ оператор $Q_{m^{\prime} m^{\prime}}$ и оператор $(20)$ умножения на $P_{s}(u)$ сохраняют индекс $m^{\prime}$, а образы $Q_{m^{\prime}, m^{\prime} \pm 1} \Theta_{a, m^{\prime} \pm 1}$ подобны $\Theta_{a m^{\prime}}$ [4],

$$
\begin{gathered}
{\left[Q_{m^{\prime} m^{\prime}}(\theta)-a(a+1)\right] \Theta_{a m^{\prime}}(u)=0, \quad Q_{m^{\prime} m^{\prime \prime}}(\theta) \Theta_{a m^{\prime \prime}}(u)=q_{m^{\prime} m^{\prime \prime}}^{a} \Theta_{a m^{\prime}}(u),} \\
q_{m^{\prime} m^{\prime \prime}}^{a} \equiv\left[a(a+1)-m^{\prime} m^{\prime \prime}\right]^{1 / 2}, \quad m^{\prime \prime}=m^{\prime} \pm 1 .
\end{gathered}
$$

Поэтому при данном $n$ каждое уравнение системы $(71)\left(m^{\prime}=\mu(\sigma), \ldots, \ell\right)$ заменой всех функций $\Psi_{m^{\prime \prime}}^{n^{\prime} \varepsilon}, n^{\prime}=0, \ldots, n+2, m^{\prime \prime}=m^{\prime}, m^{\prime} \pm 1$, их анзацами (60) сводится к уравнению, содержашему функции $\Theta_{a m^{\prime \prime}}$ с разными $a$, но одним и тем же $m^{\prime \prime}$, равным $m^{\prime}$. Так как такие функции линейно независимы, то рассматриваемое уравнение имеет решение тогда и только тогда, когда коэффициент перед каждой из них равен нулю, т.е. когда для любого допустимого $a$

$$
\begin{aligned}
& {\left[\ell(\ell+1)-2 m^{\prime 2}-(n+2)(n+3)\right] \Psi_{a m^{\prime}}^{n+2, \varepsilon}(y)-} \\
& \quad-\sum_{m^{\prime \prime}=m^{\prime} \pm 1} \gamma_{m^{\prime} m^{\prime \prime}}^{\ell \sigma} q_{m^{\prime} m^{\prime \prime}}^{a} \Psi_{a m^{\prime \prime}}^{n+2, \varepsilon x}(y)=-q \Psi_{a m^{\prime}}^{n+1, \varepsilon x}(y)-h_{a m^{\prime}}^{x}(y) \Psi_{a m^{\prime}}^{n \varepsilon x}(y)- \\
& \quad-\sum_{p=1}^{n} \sum_{s=0}^{p}(-1)^{s} V^{p s}(y) \sum_{a^{\prime}=m^{\prime}}^{\ell+n-p-\mu(\sigma)} C_{s 0 a 0}^{a^{\prime} 0} C_{s 0 a^{\prime} m^{\prime}}^{a m^{\prime}} \Psi_{a^{\prime} m^{\prime}}^{n-p, \varepsilon x}(y)
\end{aligned}
$$

где $\Psi_{a m^{\prime}}^{n \varepsilon x} \equiv 0$ при $n<0$ или $a<m^{\prime}$ или же при $a>\ell+n-\mu(\sigma)$ и, как следует из соотношений $(44),(72)$ и $(74)$,

$$
h_{a m^{\prime}}^{x}(y) \equiv\left\langle\Theta_{a m^{\prime}}(u)\left|h_{m^{\prime}}^{x}(y, \theta)\right| \Theta_{a m^{\prime}}(u)\right\rangle=h_{a}(y) .
$$

Таким образом, цепочка дифференциальных уравнений (71) сводится к рекуррентной по индексу $n$ цепочке систем линейных уравнений (75) для искомых $\Theta$-компонент $\Psi_{a m^{\prime}}^{n \varepsilon x}$ рядов (60). Эти системы нумеруются индексами $n$ и $a$. Система с данными $n$ и $a$ состоит из уравнений для неизвестных компонент $\Psi_{a m^{\prime}}^{n+2, \varepsilon x}$, причем $m^{\prime}=\mu(\sigma), \ldots, \ell$, если $a>\ell$, и $m^{\prime}=a, \ldots, \ell$, если $a \leqslant \ell$. Матрица такой системы трехдиагональна, а ее неизвестные зацеплены по индексу $m^{\prime}$, что сушественно затрудняет их нахождение в явном виде. Ключевыми для построения решения $F_{a m^{\prime}}^{n+2, x}, m^{\prime} \leqslant a$, однородной системы с такой 
матрицей являются известные связи [3] для коэффициентов Клебша-Гордана с индексами проекций моментов, отличающихся на единицу, и следуюший из представлений (61) и (64) анзац

$$
F_{a m^{\prime}}^{n+2, x}(y)=T_{a, n+2}^{\ell m^{\prime}} f_{a}^{n+2, x}(y), \quad f_{a}^{n+2, x}(y) \equiv \Psi_{a, n+2}^{n+2, \varepsilon}(y), \quad m^{\prime} \leqslant a .
$$

Обшие решения $\Psi_{a m^{\prime}}^{n \varepsilon x}(y), n \leqslant 3$, цепочки (75) выглядят не столь сложно, что позволяет по формулам (60) выразить компоненты $\Psi_{m^{\prime}}^{n \varepsilon x}(y, \theta), n \leqslant 3$, рядов (58) с $m^{\prime}=\mu(\sigma), \ldots, \ell$ через неопределенные функции $f_{a}^{n x}(y)$, известные операторы $h_{a m^{\prime}}^{x}(y)$ и функции $V^{p s}(y)$ и $\Theta_{a m^{\prime}}(u)$,

$$
\begin{aligned}
\Psi_{m^{\prime}}^{0 \varepsilon x}= & T_{\ell 0}^{\ell m^{\prime}} f_{\ell}^{0 x} \Theta_{\ell m^{\prime}}, \quad \Psi_{m^{\prime}}^{1 \varepsilon x}=\frac{q}{2} \Psi_{m^{\prime}}^{0 \varepsilon x}+\sum_{a=|\ell-1|}^{\ell+1} T_{a 1}^{\ell m^{\prime}} f_{a}^{1 x} \Theta_{a m^{\prime}}, \\
\Psi_{m^{\prime}}^{2 \varepsilon x}= & \frac{1}{12}\left(2 h_{\ell m^{\prime}}^{x}+q^{2}\right) \Psi_{m^{\prime}}^{0 \varepsilon x}+\frac{q}{4} \sum_{a=|\ell-1|}^{\ell+1} T_{a 1}^{\ell m^{\prime}} f_{a}^{1 x} \Theta_{a m^{\prime}}+\sum_{a=|\ell-2|}^{\ell+2} T_{a 2}^{\ell m^{\prime}} f_{a}^{2 x} \Theta_{a m^{\prime}} \\
\Psi_{m^{\prime}}^{3 \varepsilon x}= & \frac{1}{144}\left[q\left(8 h_{\ell m^{\prime}}^{x}+q^{2}\right)+12 V^{10}\right] \Psi_{m^{\prime}}^{0 \varepsilon x}+ \\
& +\frac{1}{40} \sum_{a=|\ell-1|}^{\ell+1}\left(4 h_{\ell m^{\prime}}^{x}+q^{2}\right) T_{a 1}^{\ell m^{\prime}} f_{a}^{1 x} \Theta_{a m^{\prime}}-\frac{V^{11}}{10 \sqrt{3}} f_{\ell}^{0 x} \sum_{a=|\ell \pm 1|} C_{\ell 010}^{a 0} T_{a 1}^{\ell m^{\prime}} \Theta_{a m^{\prime}}+ \\
& +\frac{q}{6} \sum_{a=|\ell-2|}^{\ell+2} T_{a 2}^{\ell m^{\prime}} f_{a}^{2 x} \Theta_{a m^{\prime}}+\sum_{a=|\ell-3|}^{\ell+3} T_{a 3}^{\ell m^{\prime}} f_{a}^{3 x} \Theta_{a m^{\prime}} .
\end{aligned}
$$

Используя эти представления, получаем следующие асимптотические формулы для ряда (58):

$$
\begin{aligned}
\Psi_{m^{\prime}}^{\varepsilon x}(x, y, \theta)= & {\left[1+\frac{q x}{2}+\frac{x^{2}}{12}\left(2 h_{\ell m^{\prime}}^{x}(y)+q^{2}\right)\right] \Psi_{m^{\prime}}^{\varepsilon x}(0, y, \theta)+} \\
& +\frac{x^{3}}{144}\left[q\left(8 h_{\ell m^{\prime}}^{x}(y)+q^{2}\right)+12 V^{10}\right] \Psi_{m^{\prime}}^{\varepsilon x}(0, y, \theta)+ \\
& +x^{2} \sum_{a=\ell,|\ell \pm 2|}\left(1+\frac{q x}{6}\right) T_{a 2}^{\ell m^{\prime}} f_{a}^{2 x}(y) \Theta_{a m^{\prime}}(u)+ \\
& +x \sum_{a=|\ell \pm 1|}\left[1+\frac{q x}{4}+\frac{x^{2}}{40}\left(4 h_{a m^{\prime}}^{x}(y)+q^{2}\right)\right] T_{a m^{\prime}}^{\ell m^{\prime}} f_{a}^{1 x}(y) \Theta_{a m^{\prime}}(u)+ \\
& +x^{3} \sum_{a=|\ell \pm 1|}\left[T_{a 3}^{\ell m^{\prime}} f_{a}^{3 x}(y)-\frac{V^{11}(y)}{10 \sqrt{3}} C_{\ell 010}^{a 0} T_{a 1}^{\ell m^{\prime}} f_{\ell}^{0 x}(y)\right] \Theta_{a m^{\prime}}(u)+ \\
& +x^{3} \sum_{a=|\ell \pm 3|} T_{a 3}^{\ell m^{\prime}} f_{a}^{3 x} \Theta_{3 m^{\prime}}(u)+O\left(x^{4}\right), \quad \sigma=(-1)^{\ell}
\end{aligned}
$$




$$
\begin{aligned}
\Psi_{m^{\prime}}^{\varepsilon x}(x, y, \theta)= & x\left[1+\frac{q x}{4}+\frac{x^{2}}{40}\left(4 h_{\ell m^{\prime}}^{x}(y)+q^{2}\right)\right] \Psi_{m^{\prime}}^{\varepsilon x}(0, y, \theta)+ \\
& +x^{2} \sum_{a=|\ell \pm 1|}\left(1+\frac{q x}{6}\right) T_{a 2}^{\ell m^{\prime}} f_{a}^{2 x}(y) \Theta_{a m^{\prime}}(u)+ \\
& +x^{3} \sum_{a=\ell,|\ell \pm 2|} T_{a 3}^{\ell m^{\prime}} f_{3}^{3 x}(y) \Theta_{a m^{\prime}}(u)+O\left(x^{4}\right), \quad \sigma=(-1)^{\ell+1}
\end{aligned}
$$

При $x=0$ производные асимптотик (76) $\partial_{x}^{n} \Psi_{m^{\prime}}^{\varepsilon x}=(n !) \Psi_{m^{\prime}}^{n \varepsilon x}, \quad n=0,1,2,3$, содержат разное число неизвестных функций $f_{a}^{n^{\prime} x}, n^{\prime} \leqslant n$. Поэтому линейной комбинации производных разных порядков, не содержашей ни одной функции $f_{a}^{n^{\prime} x}$, не существует. Для проекций $\partial_{x}^{n} \Psi_{a m^{\prime}}^{\varepsilon x}$ производных $\partial_{x}^{n} \Psi_{m^{\prime}}^{\varepsilon x}$ на функции $\Theta_{a m^{\prime}}(u)$ такие комбинации, т.е. связи (9), сушествуют. Например, в случае $a=\ell$ при $\sigma=(-1)^{\ell}$ и $n=0$ или $\sigma=(-1)^{\ell+1}$ и $n=1$ имеем

$$
\begin{gathered}
\partial_{x}^{n}\left(2 \partial_{x}-q\right) \Psi_{\ell m^{\prime}}^{\varepsilon x}(x, y, \theta)=0, \\
{\left[12 \partial_{x}^{2+n}\left(2 \partial_{x}-q\right)-q\left(4 h_{\ell m^{\prime}}^{x}(y)-q^{2}\right)-(12+20 n) V^{10}\right] \Psi_{\ell m^{\prime}}^{\varepsilon x}(x, y, \theta)=0 .}
\end{gathered}
$$

Отметим, что асимптотическая формула (13), доказанная в работе [6], следует из разложения (76) при $\sigma=(-1)^{\ell}$. Чтобы в этом убедиться, в $(76)$ нужно положить $\mathbf{x}=\mathbf{x}_{3}$ и $q=z_{1} z_{2} \sqrt{2 \mu_{12}}$, затем по формулам (1) выразить $\mathbf{x}_{3}$ и $\mathbf{y}_{3}$ через $\mathbf{R}$ и $\boldsymbol{\rho}$ и, наконец, оставить слагаемые, убывающие медленнее, чем $O\left(R^{2}\right)$.

Пусть теперь $t=y$, т.е. выбрана система $S^{y}$. Все построения в этом случае проводим в той же последовательности, что и в случае $t=x$. Сначала действием проектора (54) с $t=y$ на систему (25)-(27) подчиним неизвестные $\Psi_{m^{\prime}}^{n \varepsilon y}$ ряда (58) дифференциальным уравнениям

$$
\begin{aligned}
& {\left[Q_{m^{\prime} m^{\prime}}(\theta)-(n+2)(n+3)\right] \Psi_{m^{\prime}}^{n+2, \varepsilon y}(y, \theta)=-q \Psi_{m^{\prime}}^{n+1, \varepsilon y}(y)+} \\
& \quad+y^{-2} \sum_{m^{\prime \prime}=m^{\prime} \pm 1} \gamma_{m^{\prime} m^{\prime \prime}}^{\ell \sigma} Q_{m^{\prime} m^{\prime \prime}}(\theta) \Psi_{m^{\prime \prime}}^{n \varepsilon y}(y, \theta)- \\
& \quad-h_{m^{\prime}}^{y}(y, \theta) \Psi_{m^{\prime}}^{n \varepsilon y}(y, \theta)-\sum_{p=1}^{n} \sum_{s=0}^{p} V^{p s}(y) P_{s}(u) \Psi_{m^{\prime}}^{n-p, \varepsilon y}(y, \theta)
\end{aligned}
$$

где $\Psi_{m^{\prime}}^{n \varepsilon y} \equiv 0$ при $n<0$ или $n<m^{\prime}=\mu(\sigma), \ldots, \ell, Q_{m^{\prime} m^{\prime \prime}}-$ операторы $(73)$, а $h_{m^{\prime}}^{y}-$ оператор $h(\mathbf{y})($ см. $(24))$ в обкладках одинаковых функций $D_{m m^{\prime}}^{\ell \sigma *}\left(\omega^{y}\right)$,

$$
h_{m^{\prime}}^{y}(y, \theta) \equiv-y^{2} \partial_{y}\left(y^{2} \partial_{y}\right)+y^{-2} Q_{m^{\prime} m^{\prime}}(\theta)+y^{-2}\left[\ell(\ell+1)-2 m^{\prime 2}\right]+V^{00}(y)-E .
$$

Подстановкой (62) сведем уравнения (78) к алгебраическим уравнениям

$$
\begin{aligned}
& {[b(b+1)-(n+2)(n+3)] \Psi_{b m^{\prime}}^{n+2, \varepsilon y}(y)=-q \Psi_{b m^{\prime}}^{n+1, \varepsilon y}(y)+} \\
& \quad+y^{-2} \sum_{m^{\prime \prime}=m^{\prime} \pm 1} \gamma_{m^{\prime} m^{\prime \prime}}^{\ell \sigma} q_{m^{\prime} m^{\prime \prime}}^{b} \Psi_{b m^{\prime \prime}}^{n \varepsilon y}(y)-h_{b m^{\prime}}^{y}(y) \Psi_{b m^{\prime}}^{n \varepsilon}(y)- \\
& \quad-\sum_{p=1}^{n} \sum_{s=0}^{p}(-1)^{s} V^{p s}(y) \sum_{b^{\prime}=m^{\prime}}^{n-p} C_{s 0 b 0}^{b^{\prime} 0} C_{s 0 b^{\prime} m^{\prime}}^{b m^{\prime}} \Psi_{b^{\prime} m^{\prime}}^{n-p y}(y),
\end{aligned}
$$


где $m^{\prime}=\mu(\sigma), \ldots, \min (b, n+2), \Psi_{b m^{\prime}}^{n \varepsilon y}=0$, если $n<0$ или $m^{\prime}>b$ или же $n>b$, a $h_{b m^{\prime}}^{y}$ оператор $h_{m^{\prime}}^{y}$ в обкладках одинаковых функций $\Theta_{b m^{\prime}}(\theta)$,

$$
h_{b m^{\prime}}^{y}(y) \equiv-y^{-2} \partial_{y}\left(y^{2} \partial_{y}\right)+y^{-2}\left[\ell(\ell+1)+b(b+1)-2{m^{\prime}}^{2}\right]+V^{00}(y)-E .
$$

При данных $n$ и $b$ неизвестные $\Psi_{b m^{\prime}}^{n+2, \varepsilon y}, m^{\prime}=\mu(\sigma), \ldots, \min (b, n+2)$, системы (79), в отличие от решений $\Psi_{a m^{\prime}}^{n+2, \varepsilon y}$ уравнений (75), не зацеплены по индексу $m^{\prime}$. Нетривиальное решение $F_{b m^{\prime}}^{n+2, y}, m^{\prime}=\mu(\sigma), \ldots, \min (b, n+2)$, соответствуюшей однородной системы сушествует только при $b=n+2$ и элементарно выражается $\left(F_{b m^{\prime}}^{n+2, y}=f_{m^{\prime}}^{n+2, y}\right)$ через произвольные функции $f_{m^{\prime}}^{n+2, y}(y)$, равные линейным комбинациям (64) произвольных бисферических компонент $\Psi_{a, n+2}^{n+2, \varepsilon}$. По указанным причинам построение обшего решения $\Psi_{b m^{\prime}}^{n+2, \varepsilon y}, m^{\prime} \leqslant b \leqslant n+2$, цепочки систем (79) в порядке возрастания $n$ и убывания $b$ при каждом $n$ не вызывает затруднений:

$$
\begin{gathered}
\Psi_{n+2, m^{\prime}}^{n+2, \varepsilon y}(y)=f_{m^{\prime}}^{n+2, y}(y), \\
\Psi_{n+1, m^{\prime}}^{n+2, \varepsilon y}(y)=\frac{q}{2 n+4} f_{m^{\prime}}^{n+1, y}(y), \\
\Psi_{n m^{\prime}}^{n+2, \varepsilon y}(y)=\frac{1}{4 n+6}\left[\left(h_{n m^{\prime}}^{y}(y)+\frac{q^{2}}{2 n+2}\right) f_{m^{\prime}}^{n y}(y)-\right. \\
-y^{-2} \sum_{m^{\prime \prime}=m^{\prime} \pm 1} \gamma_{m^{\prime} m^{\prime \prime}}^{\ell \sigma} q_{m^{\prime} m^{\prime \prime}}^{n} f_{m^{\prime \prime}}^{n y}(y)- \\
-C_{10 n 0}^{n-10} C_{10 n-1 m^{\prime}}^{n m^{\prime}} V^{11}(y) f_{m^{\prime}}^{n-1, y}(y)+ \\
\left.+C_{20 n 0}^{n-20} C_{20 n-2 m^{\prime}}^{n m^{\prime}} V^{22}(y) f_{m^{\prime}}^{n-2, y}(y)\right]
\end{gathered}
$$

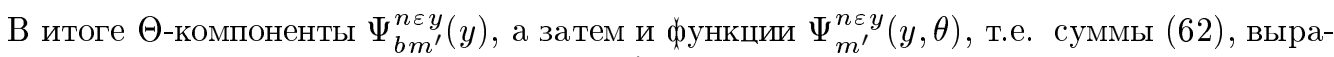
жаются через произвольные функции $f_{m^{\prime}}^{n^{\prime} y}(y) \mathrm{c} n^{\prime} \leqslant n$, известные операторы $h_{b m^{\prime}}^{y}(y)$ и функции $V^{p s}(y)$ и $\Theta_{b m^{\prime}}(u)$. Например,

$$
\begin{aligned}
\Psi_{m^{\prime}}^{0 \varepsilon y}= & f_{0}^{0 y} \Theta_{00} \delta_{m^{\prime} 0}=2^{-1 / 2} f_{0}^{0 y}, \quad \Psi_{m^{\prime}}^{1 \varepsilon y}=\frac{q}{2} \Psi_{m^{\prime}}^{0 \varepsilon y}+f_{m^{\prime}}^{1 y} \Theta_{1 m^{\prime}}, \\
\Psi_{m^{\prime}}^{2 \varepsilon y}= & \frac{1}{12}\left(2 h_{00}^{y}+q^{2}\right) \Psi_{m^{\prime}}^{0 \varepsilon y}+\frac{q}{4} f_{m^{\prime}}^{1 y} \Theta_{1 m^{\prime}}+f_{m^{\prime}}^{2 y} \Theta_{2 m^{\prime}}, \\
\Psi_{0}^{3 \varepsilon y}= & \frac{1}{144}\left[q\left(8 h_{00}^{y}+q^{2}\right)+12 V^{10}\right] \Psi_{m^{\prime}}^{0 \varepsilon y}+\frac{q}{6} f_{0}^{2 y} \Theta_{20}+f_{0}^{3 y} \Theta_{30}+ \\
& +\frac{1}{40}\left[\left(4 h_{10}^{y}+q^{2}\right) \Psi_{m^{\prime}}^{0 \varepsilon y}-4 y^{-2} \gamma_{01}^{\ell \sigma} q_{01}^{1} f_{1}^{1 y}+\frac{4}{\sqrt{3}} V^{11} \Psi_{m^{\prime}}^{0 \varepsilon y}\right] \Theta_{10}, \\
\Psi_{1}^{3 \varepsilon y}= & \frac{1}{40}\left[\left(4 h_{11}^{y}+q^{2}\right) f_{1}^{1 y}-4 y^{-2} \gamma_{01}^{\ell \sigma} q_{01}^{1} f_{0}^{1 y}\right] \Theta_{11}+\frac{q}{6} f_{1}^{2 y} \Theta_{21}+f_{1}^{3 y} \Theta_{31}, \\
\Psi_{2}^{3 \varepsilon y}= & \frac{q}{6} f_{2}^{2 y} \Theta_{22}+f_{2}^{3 y} \Theta_{32}, \quad \Psi_{3}^{3 \varepsilon y}=f_{3}^{3 y} \Theta_{33},
\end{aligned}
$$


где $f_{0}^{n y}(y) \equiv 0$ при любом $n$, если $\sigma=(-1)^{\ell+1}$. Используя эти представления, находим следуюшие асимптотики сумм (58), т.е. $D$-компонент $\Psi_{m^{\prime}}^{\varepsilon y}$ :

$$
\begin{aligned}
& \Psi_{0}^{\varepsilon y}(x, y, \theta)=\left\{1+\frac{q x}{2}+\frac{x^{2}}{12}\left(2 h_{00}^{y}(y)+q^{2}\right)+\right. \\
&\left.+\frac{x^{3}}{144}\left[q\left(8 h_{00}^{y}(y)+q^{2}\right)+12 V^{10}\right]\right\} f_{0}^{0 y}(y) \Theta_{00}(u)+ \\
&+x\left[1+\frac{q x}{4}+\frac{x^{2}}{40}\left(4 h_{00}^{y}(y)+q^{2}\right)\right] f_{0}^{1 y}(y) \Theta_{10}(u)+ \\
&+\frac{x^{3}}{10 \sqrt{3} y^{2}}\left\{y^{2} V^{11}(y) f_{0}^{0 y}(y)-\right. \\
&\left.-\left[6 \ell(\ell+1)\left(1+\sigma(-1)^{\ell}\right)\right]^{1 / 2} f_{1}^{1 y}(y)\right\} \Theta_{10}(u)+ \\
&+x^{2}\left(1+\frac{q x}{6}\right) f_{0}^{2 y}(y) \Theta_{20}(u)+x^{3} f_{0}^{3 y}(y) \Theta_{30}(u)+O\left(x^{4}\right), \\
& \Psi_{1}^{\varepsilon y}(x, y, \theta)= x\left[1+\frac{q x}{4}+\frac{x^{2}}{40}\left(4 h_{11}^{y}(y)+q^{2}\right)\right] f_{1}^{1 y}(y) \Theta_{11}(u)- \\
&-\frac{x^{3}}{5 y^{2}}[\ell(\ell+1)]^{1 / 2} f_{0}^{1 y}(y) \Theta_{11}(u)+ \\
&+x^{2}\left(1+\frac{q x}{6}\right) f_{1}^{2 y}(y) \Theta_{21}(u)+x^{3} f_{1}^{3 y}(y) \Theta_{31}(u)+O\left(x^{4}\right), \\
& \Psi_{2}^{\varepsilon y}(x, y, \theta)= x^{2}\left[1+\frac{q x}{6}\right] f_{2}^{2 y}(y) \Theta_{22}(u)+x^{3} f_{2}^{3 y}(y) \Theta_{32}(u)+O\left(x^{4}\right) .
\end{aligned}
$$

Затем из этих асимптотик выводим связи (9) при $x=0, y>0$ для проекций $\partial_{x}^{n} \Psi_{b m^{\prime}}^{\varepsilon y} \equiv$ $(n !) \Psi_{b m^{\prime}}^{\varepsilon y}$ производных $\partial_{x}^{n} \Psi_{m^{\prime}}^{\varepsilon y}$ на функции $\Theta_{b m^{\prime}}(u)$,

$$
\begin{gathered}
\partial_{x}^{b}\left(2 \partial_{x}-q\right) \Psi_{b m^{\prime}}^{\varepsilon y}(x, y)=0, \quad b \geqslant m^{\prime}=0,1,2, \\
{\left[6 \partial_{x}^{2}-2 h_{00}^{y}(y)-q^{2}\right] \Psi_{00}^{\varepsilon y}(x, y)=0,} \\
{\left[24 \partial_{x}^{3}-q\left(8 h_{00}^{y}(y)+q^{2}\right)-12 V^{10}\right] \Psi_{00}^{\varepsilon y}(x, y)=0,} \\
\partial_{x}\left[20 \partial_{x}^{2}-3\left(4 h_{10}^{y}(y)+q^{2}\right)\right] \Psi_{10}^{\varepsilon y}(x, y)= \\
=4\left\{\sqrt{3} V^{11}(y) \Psi_{00}^{\varepsilon y}(x, y)-3 y^{-2}\left[2 \ell(\ell+1)\left(1+\sigma(-1)^{\ell}\right)\right]^{1 / 2} \partial_{x} \Psi_{11}^{\varepsilon y}(x, y)\right\}, \\
\partial_{x}\left[20 \partial_{x}^{2}-3\left(4 h_{11}^{y}(y)+q^{2}\right)\right] \Psi_{11}^{\varepsilon y}(x, y)=-\frac{24}{y^{2}} \sqrt{\ell(\ell+1)} \partial_{x} \Psi_{10}^{\varepsilon y}(x, y) .
\end{gathered}
$$

Осталось рассмотреть случай тождественных частищ $p_{j}$ и $p_{k}$. Вернемся к бисферическому представлению (42) решения $\Psi^{\varepsilon}$. Как следует из формул (7) и (33), физическое решение $\Psi^{\varepsilon+}=P^{+} \Psi^{\varepsilon}$ или $\Psi^{\varepsilon-}=P^{-} \Psi^{\varepsilon}$, обладающее кроме набора квантовых чисел $\varepsilon=(E, \ell, m, \sigma)$ определенной перестановочной симметрией $P^{+} \Psi^{\varepsilon+}=\Psi^{\varepsilon+}$ или $P^{-} \Psi^{\varepsilon-}=-\Psi^{\varepsilon-}$, ортогонально всем бисферическим гармоникам $\mathcal{Y}_{a b}^{\ell m}$ с нечетным или соответственно четным индексом $b$. Такую ортогональность можно обеспечить единственным способом, а именно выбрав все произвольные функции $\Psi_{a b}^{n \varepsilon}(y), b=n$, нулевыми при нечетном или четном $b$, равном $n$. Действительно, при таком выборе по построению, описанному в разделе 4 , все функции $\Psi_{a b}^{n \varepsilon}$ с $b \leqslant n$, а значит, и все бисферические 
компоненты $\Psi_{a b}^{\varepsilon}$ с нечетным или четным $b$ окажутся нулевыми. Поэтому формулы (42) станут бисферическим представлением искомого решения $\Psi^{\varepsilon+}$ или $\Psi^{\varepsilon-}$. Согласно формулам (64) функции $f_{m^{\prime}}^{n x}$ и $f_{m^{\prime}}^{n y}$ равны нулю тогда и только тогда, когда равны нулю функции $\Psi_{a b}^{n \varepsilon}(y)$ с $n=b$. Следовательно, если положить $f_{a}^{n x}(y)=f_{m^{\prime}}^{n y}(y) \equiv 0$ при всех нечетных или четных $n$, то построенные по формулам (58)-(63), (75) и (79) $D$-компоненты $\Psi_{m^{\prime}}^{\varepsilon t}$ будут $D$-компонентами $\Psi_{m^{\prime}}^{\varepsilon+, t}$ или $\Psi_{m^{\prime}}^{\varepsilon-t}$ разложения искомого решения $\Psi^{\varepsilon+}$ или $\Psi^{\varepsilon-}$, т.е.

$$
\Psi^{\varepsilon \pm}(\mathbf{x}, \mathbf{y})=P^{ \pm} \Psi^{\varepsilon}(\mathbf{x}, \mathbf{y})=\sum_{m^{\prime}=\mu(\sigma)}^{\ell} D_{m m^{\prime}}^{\ell \sigma *}\left(\omega^{t}\right) \Psi_{m^{\prime}}^{\varepsilon \pm t}(x, y, \theta) .
$$

Другой способ построения компонент $\Psi_{m^{\prime}}^{\varepsilon t \pm}$ основан на формуле

$$
\begin{aligned}
\Psi_{m^{\prime}}^{\varepsilon \pm, t}(x, y, \theta) & \equiv\left\langle D_{m m^{\prime}}^{\ell \sigma *}\left(\omega^{t}\right) \mid P^{ \pm} \Psi^{\varepsilon}(\mathbf{x}, \mathbf{y})\right\rangle= \\
& =\left[\Psi_{m^{\prime}}^{\varepsilon t}(x, y, \theta) \pm \sigma(-1)^{m^{\prime}} \Psi_{m^{\prime}}^{\varepsilon t}(x, y, \pi-\theta)\right],
\end{aligned}
$$

следуюшей из определений (8), (33) и равенств

$$
P_{j k} D_{m m^{\prime}}^{\ell}\left(\omega^{t}\right)=(-1)^{\ell} D_{m,-m^{\prime}}^{\ell}\left(\omega^{t}\right), \quad P_{j k} \theta=\pi-\theta, \quad t=x, y .
$$

Стоит отметить, что исследованные разложения (69) и (70) решения $\Psi^{\varepsilon}$ порождают аналогичные представления его фаддеевской компоненты $\Psi_{i}^{\varepsilon}$,

$$
\begin{aligned}
& \Psi_{i}^{\varepsilon}(\mathbf{x}, \mathbf{y})=\sum_{n=\mu(\sigma)}^{\infty} x^{n} \sum_{m^{\prime}=\mu(\sigma)}^{\ell} D_{m m^{\prime}}^{\ell \sigma *}\left(\omega^{x}\right) \sum_{a=m^{\prime}}^{\ell+n-\mu(\sigma)} \Psi_{i a m^{\prime}}^{n \varepsilon x}(y) \Theta_{a m^{\prime}}(u), \\
& \Psi_{i}^{\varepsilon}(\mathbf{x}, \mathbf{y})=\sum_{n=\mu(\sigma)}^{\infty} x^{n} \sum_{m^{\prime}=\mu(\sigma)}^{\ell} D_{m m^{\prime}}^{\ell \sigma *}\left(\omega^{y}\right) \sum_{b=m^{\prime}}^{n} \Psi_{i b m^{\prime}}^{n \varepsilon y}(y) \Theta_{b m^{\prime}}(u) .
\end{aligned}
$$

Чтобы убедиться в этом, сначала нужно в уравнение Фаддеева (3) для $\Psi_{i}^{\varepsilon}$ подставить $\Psi_{i}^{\varepsilon}$ в виде анзаца (83) или (84), заменить $V_{i}$ рядом (5), а $\Psi^{\varepsilon}$ - рядом (69) или (70), затем спроецировать полученное уравнение на $D$-базис (8) и вывести для искомых $\Theta$-компонент $\Psi_{i a m^{\prime}}^{n \varepsilon t}(y), t=x, y$, рекуррентные цепочки уравнений. Например, в случае $t=y$ получится цепочка

$$
\begin{gathered}
{[b(b+1)-(n+2)(n+3)] \Psi_{i b m^{\prime}}^{n+2, \varepsilon y}(y)=-q \Psi_{b m^{\prime}}^{n+1, \varepsilon y}(y)-\sum_{p=0}^{n} V_{i p} \Psi_{b^{\prime} m^{\prime}}^{n-p, \varepsilon y}(y)+} \\
+y^{-2} \sum_{m^{\prime \prime}=m^{\prime} \pm 1} \gamma_{m^{\prime} m^{\prime \prime}}^{\ell \sigma} q_{m^{\prime} m^{\prime \prime}}^{b} \Psi_{i b m^{\prime \prime}}^{n \varepsilon y}(y)-\left[h_{b m^{\prime}}^{y}(y)-V^{00}(y)\right] \Psi_{i b m^{\prime}}^{n \varepsilon}(y)
\end{gathered}
$$

разрешимость которой несложно доказать по индукции. Потом можно выразить компоненты $\Psi_{i b m^{\prime}}^{n \varepsilon y}$ через найденные компоненты $\Psi_{b m^{\prime}}^{n \varepsilon y}$ и построить асимптотику ряда (84). Это и предполагается сделать в дальнейшем. 


\section{6. ЗАКЛЮЧЕНИЕ}

Перечислим основные результаты работы. Было показано, что в случае центральных взаимодействий (5) довольно широкого класса обшее $\Psi$ и физическое $\Psi^{\varepsilon}$ регулярные решения уравнения Шредингера (2) вблизи точки парного удара являются рядами $(21),(39)$ и $(42),(69),(70)$ по целым степеням $x$. Ключевые для построения таких рядов компоненты $\Psi_{b \beta}^{n}, \Psi_{a b}^{n \varepsilon}$ и $\Psi_{b m^{\prime}}^{n \varepsilon y}$ удалось подчинить простым цепочкам алгебраических уравнений $(30),(43)$ и (79), рекуррентных по индексу $n$ и не зацепляюшихся при данном $n$ по индексу $b$, индексам $a, b$ или $b, m^{\prime}$. Предложенная редукция исходного шестимерного уравнения Шредингера (2) к таким цепочкам позволила вывести в явном виде асимптотические формулы (47)-(49) и (76), (81) бисферических $\Psi_{a b}^{\varepsilon}$ и $D$-компонент $\Psi_{m^{\prime}}^{\varepsilon t}, t=x, y$, физического решения $\Psi^{\varepsilon}$ вплоть до слагаемых $O\left(x^{4}\right)$ и $O\left(x^{3}\right)$. Как пояснялось, из этих формул следуют и ранее неизвестные связи (50)-(52), (77), (82), и соотношения (10)-(13), доказанные другими авторами [6], [7], [12]-[14], причем в частном случае чисто кулоновских парных взаимодействий (4). В заключение отметим, что выбор системы $S^{y}$ в качестве "подвижной" представляется оптимальным для построения $D$-компонент физического решения $\Psi^{\varepsilon}$ вблизи точки парного удара. После того как последовательно найдены компоненты $\Psi_{b m^{\prime}}^{n \varepsilon y}$ и $\Psi_{m^{\prime}}^{\varepsilon y}$ все отвечаюшие им в системе $S^{x}$ компоненты, в том числе и бисферические, можно найти по доказанным формулам (65)-(68).

\section{Список литературы}

[1] С. П. Меркурьев, Л. Д. Фаддеев. Квантовая теория рассеяния для систем нескольких частиц. М.: Наука, 1985

[2] V.V. Kostrykin, A. A. Kvitsinsky, S. P. Merkuriev. Few-Body Syst. 1989. V. 6. P. 97.

[3] Д. А. Вариалович, А.Н. Москалев, В.К. Херсонский. Квантовая теория углового момента. М.: Наука, 1975.

[4] А. Ф. Никифоров, В. Б. Уваров. Основы теории специальных функций. М.: Наука, 1974.

[5] С.И. Виницкий, Л. И. Пономарев. ЭЧАЯ. 1982. Т. 13. С. 1336.

[6] В.И. Коробов. ЯФ. 1989. Т. 50. С. 1595.

[7] V.I. Korobov, I. V. Puzynin, S. I. Vinitsky. Muon Catalyzed Fusion. 1992. V. 7. P. 63.

[8] Г. И. Марчук. Методы вычислительной математики. М.: Наука, 1989.

[9] P. M. Prenter. Splines and Variational Methods. N.Y.: Wiley, 1975.

[10] К. Вильдермут, Я. Тан. Единая теория ядра. М.: Мир, 1980.

[11] Ю. С. Завьялов, Б. И. Квасов, В. Л. Мирошниченко. Методы сплайн-функций. М.: Наука, 1980.

[12] T. Kato. Commun. Pure and Appl. Math. 1957. V. 10. P. 151.

[13] W. A. Bingel. Z. Naturforsh. A. 1963. V. 18. P. 1249.

[14] R. T. Pack, W. B. Brown. J. Chem. Phys. 1966. V. 45. P. 556.

[15] F. T. Smith. J. Chem. Phys. 1959. V. 31. P. 1352.

[16] В. И. Смирнов. Курс высшей математики, Т. 3. Ч. 1, 2. М.: Наука, 1974.

[17] E. S. Chang, U. Fano. Phys. Rev. A. 1972. V. 6. P. 173.

Поступила в редакцию 25.IV.2002 г., после доработки 18.XII.2002 г. 